\title{
Dysbiosis of skin microbiome and gut microbiome in melanoma progression
}

\author{
Chahrazed Mekadim', Helena Kupcova Skalnikova², Jana Cizkova ${ }^{2,3}$, Veronika Cizkova ${ }^{2,4}$, Anna Palanova ${ }^{2}$,
} Vratislav Horak $^{2}$ and Jakub Mrazek ${ }^{1 *}$

\begin{abstract}
Background: The microbiome alterations are associated with cancer growth and may influence the immune system and response to therapy. Particularly, the gut microbiome has been recently shown to modulate response to melanoma immunotherapy. However, the role of the skin microbiome has not been well explored in the skin tumour microenvironment and the link between the gut microbiome and skin microbiome has not been investigated in melanoma progression. Therefore, the aim of the present study was to examine associations between dysbiosis in the skin and gut microbiome and the melanoma growth using MeLiM porcine model of melanoma progression and spontaneous regression.

Results: Parallel analysis of cutaneous microbiota and faecal microbiota of the same individuals was performed in 8 to 12 weeks old MeLiM piglets. The bacterial composition of samples was analysed by high throughput sequencing of the V4-V5 region of the $16 \mathrm{~S}$ rRNA gene. A significant difference in microbiome diversity and richness between melanoma tissue and healthy skin and between the faecal microbiome of MeLiM piglets and control piglets were observed. Both Principal Coordinate Analysis and Non-metric multidimensional scaling revealed dissimilarities between different bacterial communities. Linear discriminant analysis effect size at the genus level determined different potential biomarkers in multiple bacterial communities. Lactobacillus, Clostridium sensu stricto 1 and Corynebacterium 1 were the most discriminately higher genera in the healthy skin microbiome, while Fusobacterium, Trueperella, Staphylococcus, Streptococcus and Bacteroides were discriminately abundant in melanoma tissue microbiome. Bacteroides, Fusobacterium and Escherichia-Shigella were associated with the faecal microbiota of MeLiM piglets. Potential functional pathways analysis based on the KEGG database indicated significant differences in the predicted profile metabolisms between the healthy skin microbiome and melanoma tissue microbiome. The faecal microbiome of MeLiM piglets was enriched by genes related to membrane transports pathways allowing for the increase of intestinal permeability and alteration of the intestinal mucosal barrier.
\end{abstract}

Conclusion: The associations between melanoma progression and dysbiosis in the skin microbiome as well as dysbiosis in the gut microbiome were identified. Results provide promising information for further studies on the local skin and gut microbiome involvement in melanoma progression and may support the development of new therapeutic approaches.

\footnotetext{
*Correspondence: mrazek@iapg.cas.cz

${ }^{1}$ Laboratory of Anaerobic Microbiology, Institute of Animal Physiology

and Genetics of the Czech Academy of Sciences, Videnska 1083, 142

20 Prague, Czech Republic

Full list of author information is available at the end of the article
} 
Keywords: Melanoma, Skin cancer, Tumour microenvironment, MeLiM, Pig, Skin microbiome, Gut microbiome, Gutskin axis, Dysbiosis, Metagenomic analysis, NGS

\section{Background}

Cutaneous Melanoma (CM) is a malignant skin cancer originating from epidermal melanocytes $[1,2]$. Even though it is less common than other skin cancers, $\mathrm{CM}$ is more lethal due to its high metastatic potential [2-4]. Considering the aggressiveness of this disease, it is important to identify the risk factors associated with melanoma development to improve diagnosis and treatment methods of this serious skin cancer. Different risk factors are associated with melanoma development: besides the genetic predisposition, such as the familial history of melanoma or other skin cancers and type of melanocytic nevi, other environmental factors, particularly the sun and UV radiation exposure, increase the risk of melanoma development [4-6].

Recently, the host microbiome is considered as a new component of the tumour microenvironment that influences tumour cell metabolism and plays a role in the cancer pathogenesis and treatment response $[7,8]$. The commensal microbes interact directly with the cancerous cells of the tumour tissue, in which they are residing. Indirect effects could occur when the tumour development is affected by the metabolites of the microbiome from another location or by the administration of probiotics in the host diet [9].

Many observations suggested that the skin commensal microbiome may promote skin immunity and confer the host defence including the protection against skin inflammatory disorders, infections, wounds and skin cancer [10-19]. Several studies have suggested a potential role of the microbiota in skin carcinogenesis [20]. A reduced rate of skin cancer was observed in germ-free rats [21]. Similarly, different microorganism-associated molecular patterns (MAMPs) were identified as the trigger of receptors responsible for the inflammatory response that leads to tumour development, while skin inflammation in response to tumour promoters was reduced in mice lacking receptors [22-24].

Several studies have investigated the gut-skin connection in various skin diseases including skin cancer [2527]. The gut-skin axis indicates that the gut microbiota and its metabolites can have a critical role in the development or prevention of skin cancers, including melanoma. In human, gut microbiota and its metabolites may have a mechanistic impact on antitumor immunity and immunotherapy in patients with advanced melanoma [28-37]. Also, it has been reported that the administration of selected strains from commensal intestinal microbiota may establish anti-tumour immunity and restrict melanoma growth in germ-free WT mice [38].

In order to investigate changes in the gut and skin microbiota composition during melanoma development, swine models are highly suitable due to the high similarities with human in terms of skin and gastrointestinal anatomy and physiology, genetics, immunology and pathophysiology of many human diseases [39-41]. The Melanoma-bearing Libechov Minipig (MeLiM) is a unique large animal model of hereditary melanoma [42]. In the MeLiM strain, the melanomas occur only in animals with pigmented skin, as the white pigs lack melanocytes in their skin. The MeLiM piglets are born with several nodular melanomas or the melanomas develop postnatally. The melanomas mostly invade into deep dermis and subcutaneous fat (corresponding to Clark level IV to $\mathrm{V}$ in human staging). At the age of 8 to 12 weeks, the spontaneous regression of melanomas starts to occur in the majority of animals, which is characterized by lymphocyte infiltration, and flattering and colour fading of tumours due to the replacement of the tumour by fibrous tissue [43-46]. However, in approximately $20 \%$ of piglets, the melanoma progression develops which is characterized by tumour growth and spread by metastases [47], mainly into the lymph nodes and lungs, cachexia and animal growth retardation. The MeLiM model enables to study the melanoma spontaneous development without any therapeutic interventions, which is not possible in human.

The aim of the present study was to assess the association between the diversity, composition and function of the skin and faecal microbiome and melanoma development and to compare the bacterial composition in such entities using high throughput sequencing. The samples were collected from multiple sites (inner melanoma tissue, melanoma surface, healthy skin and stool) of experimental piglets at different ages throughout melanoma progression and melanoma spontaneous regression (experimental scheme is presented in Fig. S1). Findings could contribute to the characterization of skin and gut microbiome composition and modification, as well as functional mechanisms following melanoma progression.

\section{Results}

Skin and stool microbiome samples were collected from MeLiM piglets with melanoma progression $(n=10)$ and spontaneous regression $(n=10)$, as well as from crossbreds of MeLiM and white strain with black skin and 
several melanomas with regressive disease course $(n=4)$ and were compared to control MeLiM $\mathrm{x}$ white crossbreds with melanoma-free white skin $(n=10)$.

A total of $13,747,282$ sequences were obtained from different samples. The mean sequence length was $275 \mathrm{bp}$. The metagenomic analysis of each microbiota (faecal and cutaneous) was performed separately.

\section{Diversity analysis}

Alpha diversity of samples collected from different sites (melanoma tissue, melanoma surface, healthy skin and stool) was evaluated to determine the bacterial diversity of each animal phenotype (progression, spontaneous regression and white control) using Chaol, ACE, observed species, Fisher, Shannon, Simpson and Inverse Simpson indexes. The results are represented in the boxplot graph (Fig. 1, S2, S3). The obtained values after comparison between different groups are reported in (Table S1, S2). A highly significant difference in the skin microbiota diversity was markedly noticed between different sites of sampling (melanoma tissue, melanoma surface and healthy skin) and between different animal groups (MeLiM, white control, and black crossbred) (KruskalWallis test; $p<0.05$ ). The highest bacterial diversity was

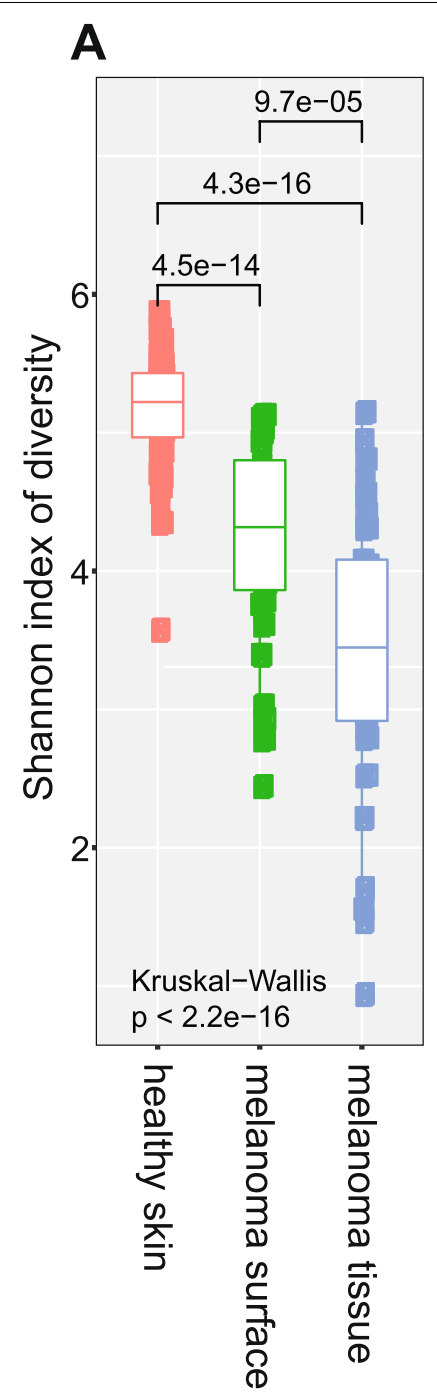

B
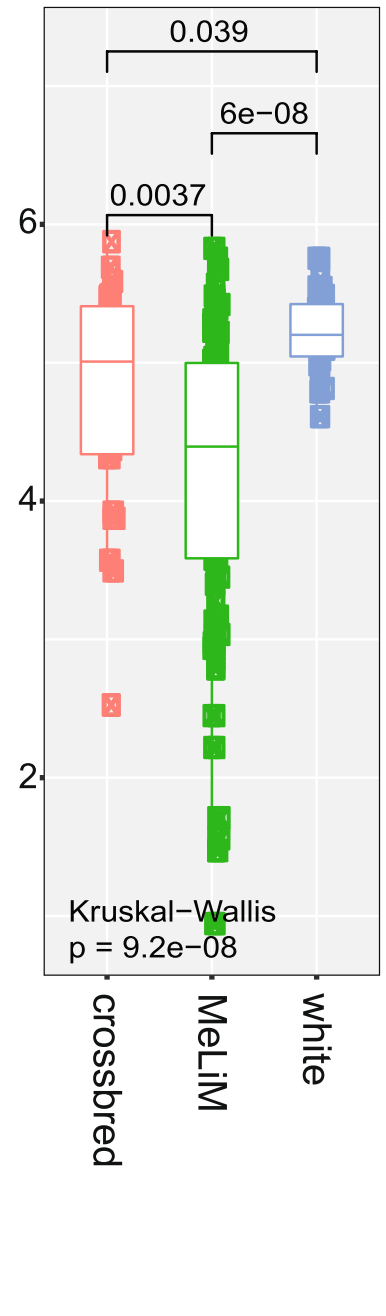
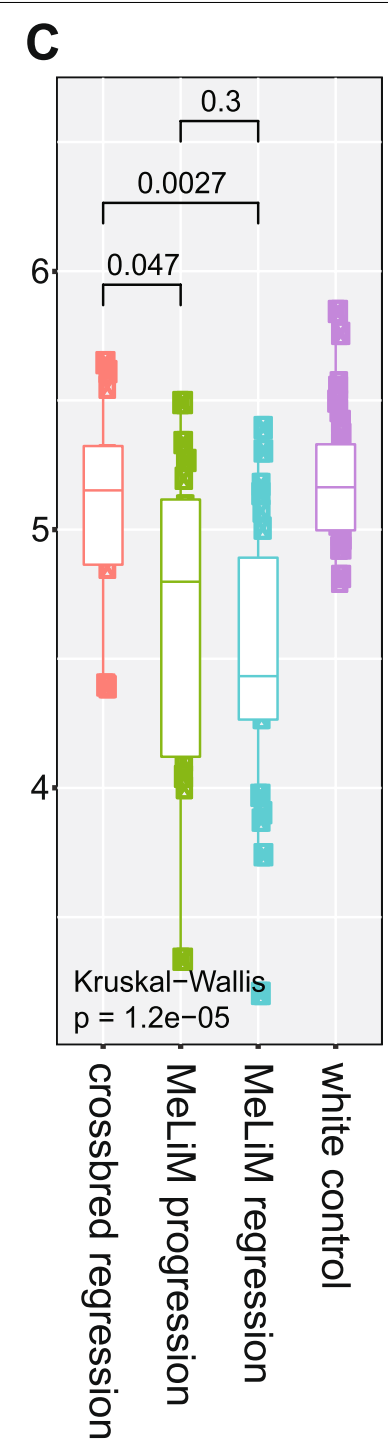

Fig. 1 Box-plot of Shannon index of diversity a) in cutaneous microbiome among different cutaneous samples (healthy skin, melanoma surface and melanoma tissue), b) cutaneous microbiome of different animal breeds (white, crossbred, MeLiM) and c) faecal microbiome of different piglets groups (white control, crossbred with melanoma regression, MeLiM with melanoma regression and MeLiM with melanoma progression) using Kruskal-Wallis pairwise test ( $p$-value $\leq 0.05$ ) was used to compare between different samples 
observed in the healthy skin microbiome of white control pigs (Fig. 1a, 1b, S2a, S2b). The diversity of melanoma surface and melanoma tissue in the MeLiM progressive group was lower than in the MeLiM with spontaneous regression but the differences were not significant (Fig. S3a). However, through the animal age, the diversity of the skin microbiome significantly increased in melanoma regression group while no distinct difference was observed in the skin microbiome of melanoma progression group between the analysed ages (Fig. S3b).

Similarly, in the faecal microbiota, the highest alpha diversity was observed in white control animals while the MeLiM animals with melanoma progression showed the lowest alpha diversity. (Fig. 1c, S2c). Also, no significant difference was observed between progressive and regressive melanoma groups, except a significant difference was noted between the faecal microbiota diversity of crossbred with melanoma regression and faecal microbiota diversity of MeLiM animals with melanoma regression (Fig. 1c, S2c). Throughout the age, the bacterial faecal community was dynamic and highly diverse in the white control animals and animals with melanoma regression (especially between ages 8 weeks and 10 weeks) whereas it was more stable in the animals with melanoma progression (Fig. S3c).

In addition, beta diversity was used to analyse the dissimilarities between bacterial communities in skin and stool samples separately. Principal Coordinate Analysis (PCoA) and Non-metric multidimensional scaling
(NMDS) plots based on Bray Curtis distance were performed to reveal disparately separated microbial communities (Fig. 2, S4). In the skin microbiome, three major clusters were distinguished according to the nature of samples (melanoma tissue, melanoma surface and healthy skin) (Fig. 2a, S4a). Clusters of the progressive melanoma group and the control healthy skin group were distinctly separated showing the higher significant differences between those two bacterial communities (Fig. 2b, S4b). The cluster of regressive melanoma is the largest one which reflects the bacterial diversity dissimilarities between samples of this categorical group, which were heterogeneous because they belong to two different animal groups (MeLiM and Crossbred) and due to the changes in microbial composition throughout the ages. The bacterial diversity of faecal samples in crossbred with melanoma regression was more similar to that in white crossbred animals (control) and MeLiM progressive and regressive groups were clustered together indicating that no distinct difference was observed in faecal bacterial structures between the progressive and regressive melanoma (Fig. 2c, S4c).

\section{Relative abundance analysis and taxonomic composition}

The relative abundance composition of cutaneous skin microbiome at the phylum level showed that the higher abundance of Firmicutes and Proteobacteria were associated with skin devoid of melanoma and white control pigs, while the relative abundance of Fusobacteria was
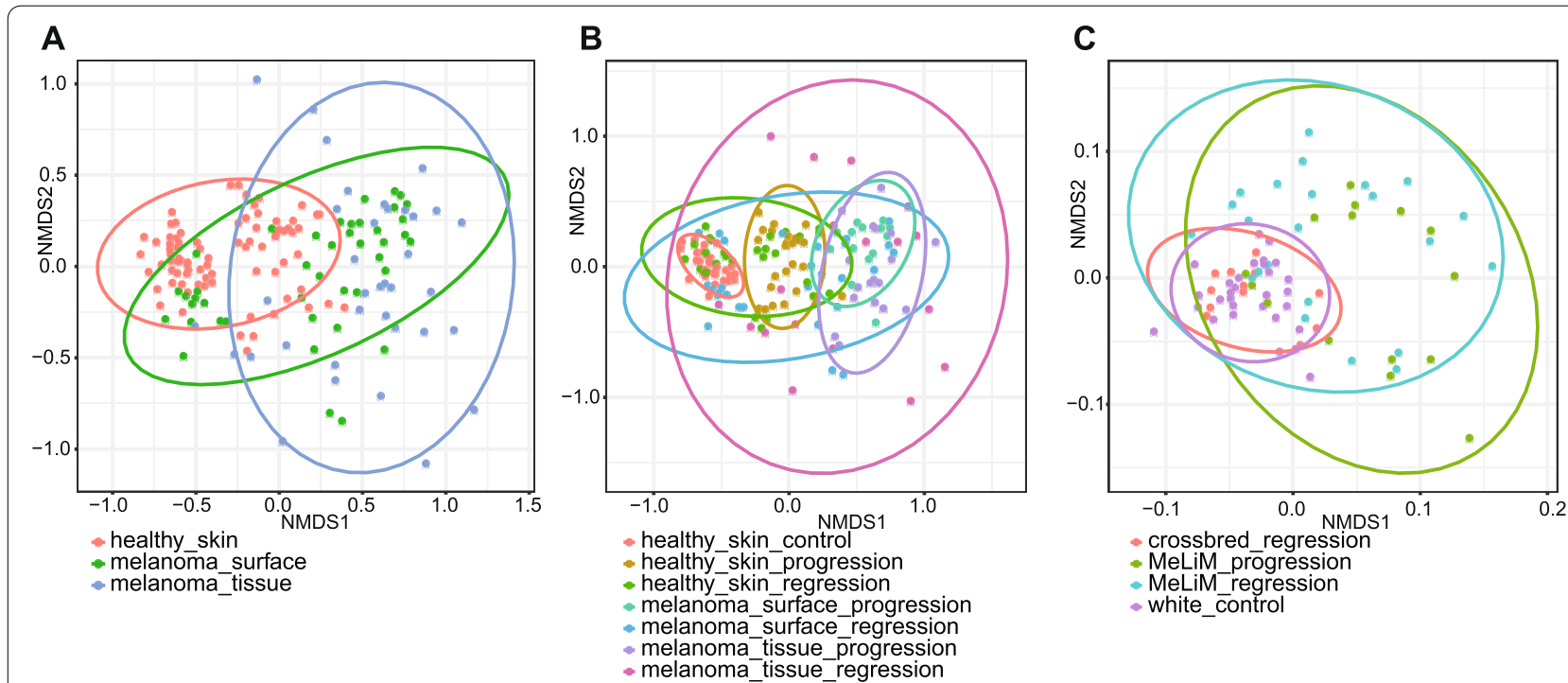

Fig. 2 Non-metric Multidimensional Scaling (NMDS) plots based on the Bray Curtis distance matrix for beta diversity comparison of bacterial communities a) in skin microbiome among different samples (healthy skin, melanoma surface and melanoma tissue) b) in skin microbiome among multiple samples in different disease conditions (control, melanoma progression and melanoma regression) and c) in faecal microbiome of different piglets groups (white control, crossbred with melanoma regression, MeLiM with melanoma regression and MeLiM with melanoma progression). The confidence level of the ellipse was 95\% 
associated with melanoma tissue and melanoma surface in MeLiM piglets with melanoma progression and spontaneous regression (Fig. 3a), (Table S3). The Firmicutes and Proteobacteria ratios were lower in those last groups. Actinobacteria and Bacteroidetes abundances were relatively similar in all samples. The relative abundances of Fusobacteria were decreased dramatically in the regressive melanoma tissue microbiome from $20.9 \%$ at the age of 8 weeks to $5.0 \%$ at the age of 12 weeks, while no significant changes were observed in the bacterial composition of progressive melanoma skin microbiome throughout the ages (Fig. 3b), (Table S3).

The bacterial phylogenetic compositions of the faecal microbiota of piglets at the phylum level were dominated by Firmicutes and Bacteroidetes, but these were slightly less abundant in MeLiM piglets (Fig. 3c), (Table S4). The higher abundance of Proteobacteria and Fusobacteria were associated with faecal microbiota in MeLiM piglets (Fig. 3c). In faecal microbiota of MeLiM piglets with melanoma regression, the relative abundances of Fusobacteria and Proteobacteria dropped from $3.7 \%$, and $18.7 \%$, respectively, at the age of 8 weeks, to $1.3 \%$, and $2.6 \%$, respectively at the age of 12 weeks, while the relative abundances of Firmicutes and Bacteroidetes increased significantly from $28.4 \%$, and $19.0 \%$, respectively, at the age of 8 weeks, to the $40.4 \%$, and $29.1 \%$, respectively, at the age of 12 weeks. In MeLiM piglets with melanoma progression, no

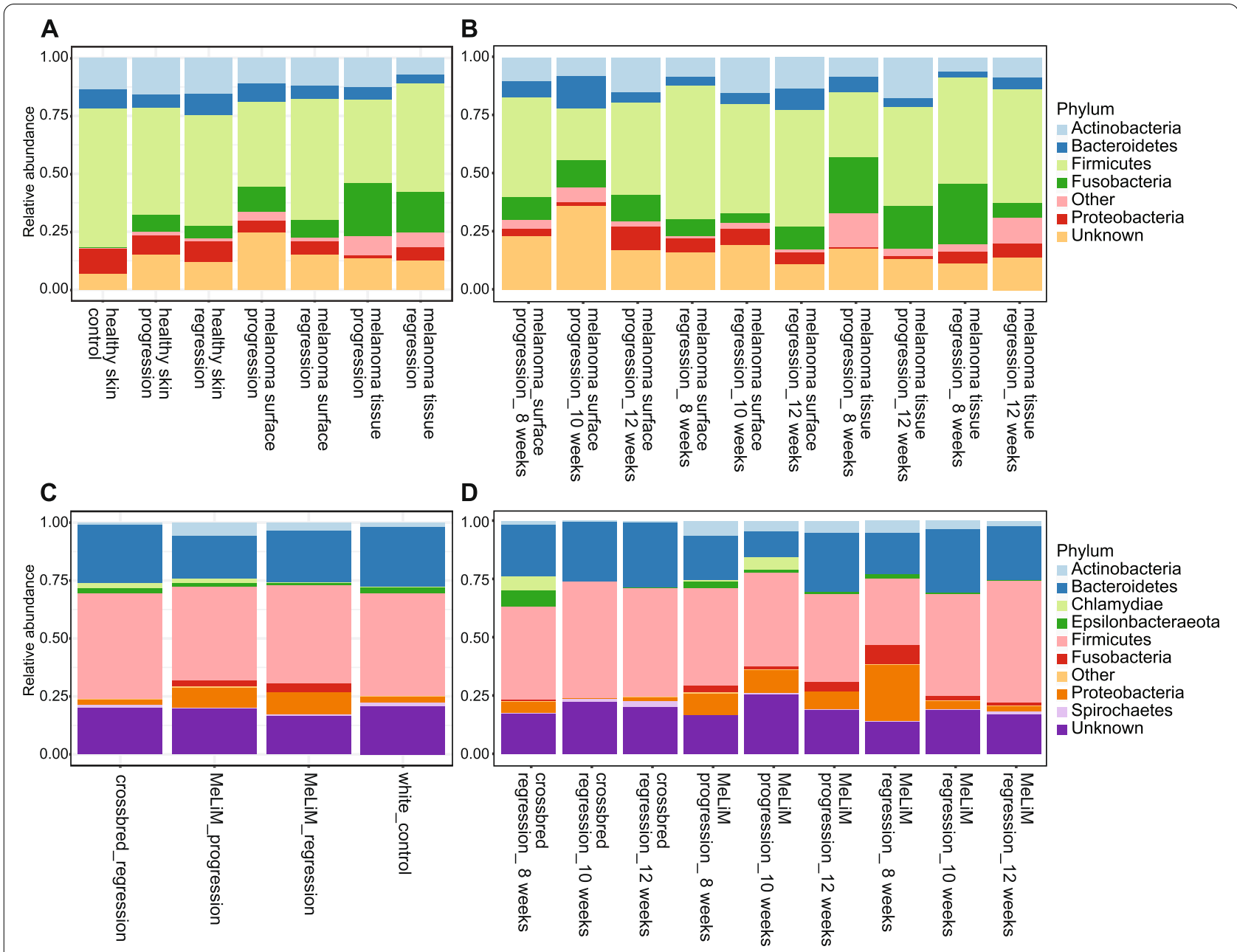

Fig. 3 Relative abundance of the microbial population at the phylum level a) in skin microbiome among different cutaneous samples (healthy skin, melanoma surface and melanoma tissue) in healthy piglets or piglets with melanoma (control, melanoma progression and melanoma regression) b) in melanoma surface and melanoma tissue microbiome with melanoma regression and melanoma progression at different ages $(8,10,12$ weeks), $\mathbf{c})$ in faecal microbiome of different piglets groups (white control, crossbred with melanoma regression, MeLiM with melanoma regression and MeLiM with melanoma progression) and $\mathbf{d}$ ) in faecal microbiome of piglets with melanoma (crossbred with melanoma regression, MeLiM with melanoma regression and MeLiM with melanoma progression) at different (8, 10, 12 weeks) 
significant changes were observed in the bacterial composition of faecal microbiota throughout the monitored ages (Fig. 3d), (Table S4).

At the genus level, the microbiome of skin devoid of melanoma in white piglets was dominated by Clostridium sensu stricto 1, Corynebacterium 1, and Lactobacillus while the microbiome of melanoma tissue in the MeLiM piglets was dominated by Fusobacterium, Staphylococcus, Trueperella and Streptococcus (Table S3). The faecal microbiota of crossbred piglets was dominated by Clostridium sensu stricto 1, Lactobacillus, Prevotella 9, Ruminococcus and Faeclibacterium. The relative abundance of Bacteroides was significantly higher in the faecal microbiota of MeLiM piglets with melanoma progression (Table S4).
The linear discriminant analysis (LDA) effect size (LEfSe) results (Fig. 4, S5) at genus level in skin microbiome showed that Fusobacterium, Staphylococcus, Trueperella, Streptococcus, Peptostreptococcus and Peptoniphilus were the more notable genera associated with melanoma microbiome and Clostridium sensu stricto 1, Corynebacterium 1, Lactobacillus, Turicibacter, Terrisporobacter and Enterococcus were bacterial genera most associated with healthy skin microbiome (Fig. S5a, S5b). Skin microbiome of piglets with progressive melanoma was related with Fusobacterium, Trueperella, Bacteroides, and Porphyromonas compared to the skin microbiome of piglets with regressive melanoma, where Enterococcus, Acinetobacterium, Bifidobacterium, Lactobacillus and Prevotella 9 were discriminately abundant

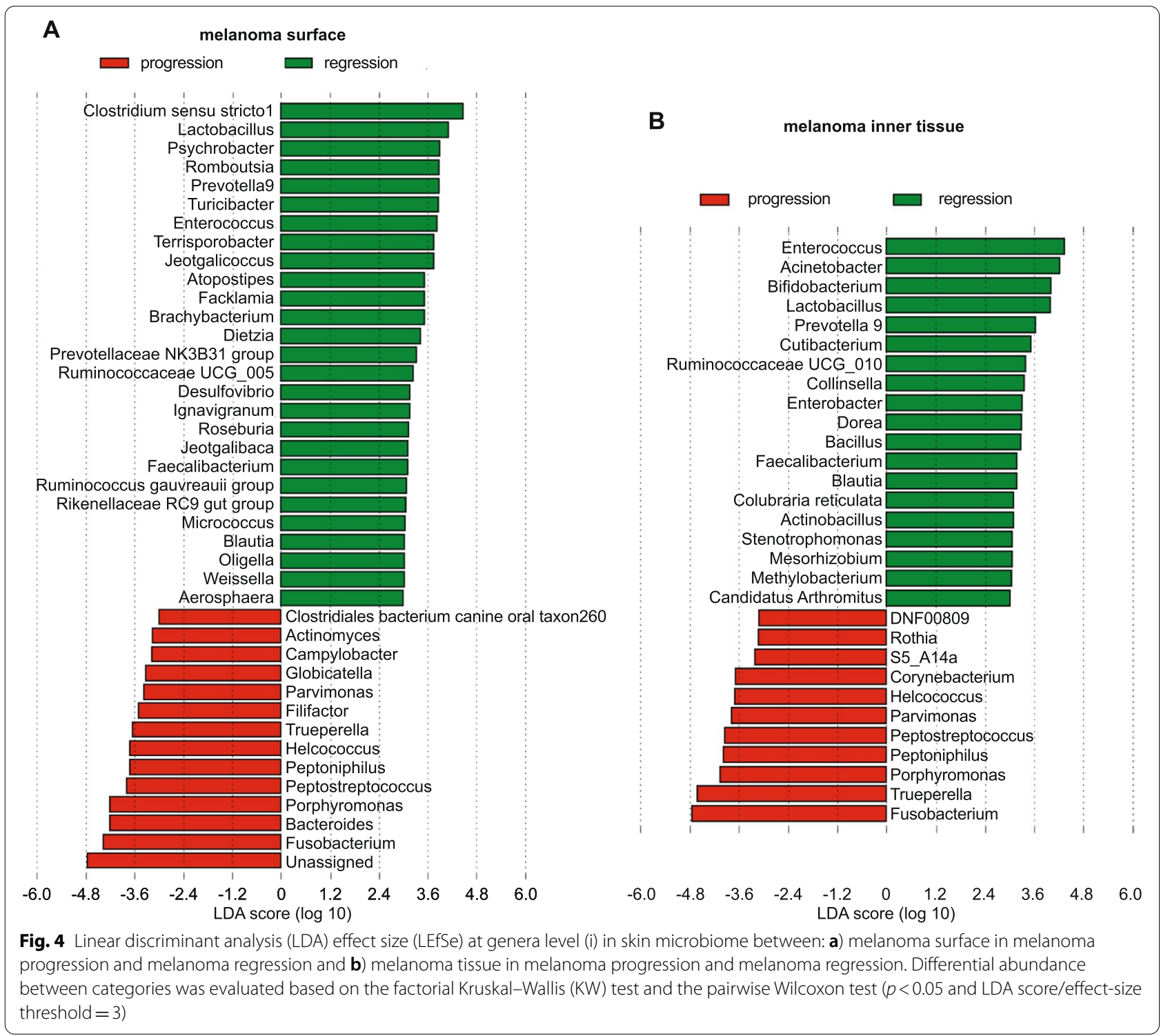


(Fig. 4a, b). In the faecal microbiome, LDA revealed a discriminant abundance of Bacteroides and Fusobacterium in MeLiM piglet with melanoma progression and Escherichia-Shigella in MeLiM piglets with melanoma regression (Fig. S5c). Bacteroides, Escherichia-Shigella and Fusobacterium were the most discriminant biomarkers in the faecal microbiome of MeLiM piglets while Prevotella 9, Lactobacillus and Faecalibacterium were the most discriminately abundant genera in the faecal microbiota of crossbred piglets (Fig. S5d).

These differences in relative abundances of certain genera reflect the dissimilarities in the skin and faecal microbiome between multiple experimental categories (melanoma tissue vs healthy skin, melanoma regression vs melanoma progression and MeLiM piglets vs crossbred piglets).

\section{Functional pathways analysis}

To predict the potential function profiles of the skin and gut microbiota in piglets with melanoma progression or melanoma regression, the relative abundances of KEGG pathways were explored based on PICRUSt analysis. A total of 248 and 130 unique KEGG Orthology (KO) pathways at level 3 were predicted in the skin microbiome and faecal microbiome respectively (Table S5, S6).

At the second level of KEGG pathways, forty differently abundant pathways indicated a significant difference in the functions of the melanoma tissue microbiome compared with the healthy skin microbiome (Fig. S6a, S7a). Nineteen pathways were enriched in melanoma tissue microbiome, including Membrane transport, Translation, Glycan biosynthesis and metabolism, Nucleotide metabolism, Metabolism of cofactors and vitamins, Other ion-coupled transporters, Replication, Recombination and repairs of proteins, Infectious diseases, Protein folding and associated processing, Signalling molecules and interaction, Membrane and intracellular structural molecules, Amino acid metabolism, Translation proteins, Cell growth and death, Cell motility and secretion, Electron transfer carries, Digestive system and immune system diseases. There were eight most significant pathways associated with healthy skin microbiome including Amino acid metabolism, Xenobiotics biodegradation and metabolism, Carbohydrate metabolism, Sporulation, Lipid metabolism, Transcription, Metabolism of other amino acids, Energy metabolism in addition to pathways in cancers. Twenty-four KEGG pathways showed a significant difference in the potential function of melanoma tissue microbiome between melanoma regression and melanoma progression, fourteen of them were significantly enriched in progressive melanoma tissue microbiome, such as Replication and repair, Translation, Nucleotide metabolism, Metabolism of cofactors and vitamins, Metabolism of terpenoids and polyketides, Folding, sorting and degradation, Protein folding and association processing, Enzymes families. The relative abundance of Cell motility, Signal transduction, Sporulation, Biosynthesis and biodegradation of secondary metabolites pathways were significantly abundant in the regressive melanoma tissue microbiome (Fig. S6b, S7b).

In the faecal microbiome, twenty-eight KEGG pathways at level 2 revealed significant differences in potential functions between MeLiM and crossbred piglets (Fig. S6c, S7c). Membrane transport, Carbohydrate metabolism and Xenobiotics biodegradation and metabolism were the principal significant abundant functions associated with MeLiM faecal microbiota. Replication and repair, Translation, Cell motility, Amino acid metabolism, Nucleotide metabolism and Energy metabolism were the most significant pathways associated with the faecal microbiota of crossbred piglets. In the faecal microbiota of MeLiM piglets, five KEGG pathways at level 3 detected a significant difference in predicted functional profiles between regressive melanoma and progressive melanoma. Fatty acids biosynthesis and Lipid biosynthesis metabolism were significantly associated with progressive melanoma, while Selenocompound metabolism, Cell division (unclassified function) and General function prediction (unclassified) were the significant abundant pathways related to regressive melanoma (Fig. S6d, S7d). The results have shown that in addition to community differences, there may be differences in the functionalities of the microbiome between MeLiM piglets and healthy controls.

\section{Discussion}

Several studies have reported the association of commensal microbiota of human or animal models with cancers, mostly focused on colorectal cancer [48-51], in addition to gastric [52, 53], liver [54], pancreatic [55, 56], lung [57-59], breast [60, 61] and bladder [62, 63] cancers. Generally, many reports suggested that microbiota induces carcinogenesis and other ones supported that microbiota play protective roles against cancer [64-67]. Recent reviews focused on the importance of the microbiome in skin cancer research and explored the crosstalk between the immune system and the skin microbiota in health and diseases (including cancer) $[6,11,12,68]$. The profound reliance of the skin immune system on its resident microbiota for both host defence and tissue repair led to the integration of the skin microbiota and its metabolites as an intrinsic regulator of immune responses in the tissue microenvironments [13, 69-71]. The interactions between skin immune cells and microbiota are not only within the local microenvironment but also the skin immune system was stimulated by 
metabolites of microbes from other body sites (e.g. gastrointestinal tract) [9]. The gut microbiota is involved in cancer and is associated with anticancer therapy efficacy. Recently, several studies illustrated that the gut microbiota and the treatment with faecal microbiota transplantation (FMT) promoted the responses to anti-PD-1 immunotherapy and restored the tumour microenvironment in patients with advanced melanoma [28-30, 32, $34,35,72,73]$.

In this study, the association between melanoma development and the changes in the bacterial composition of the gut and the skin microbiome were explored. The samples were collected from melanoma tissue, melanoma surface and healthy skin of porcine models: MeLiM piglets with melanoma progression or melanoma spontaneous regression and crossbred piglets with melanoma spontaneous regression or healthy (white) controls. Pigs are valuable large animal model due to similar anatomy and physiology, including metabolism and nutritional requirements to human. Human and pigs have been previously shown to share the major bacterial phyla (Firmicutes and Bacteroidetes) in both their gut and skin microbiome. Nonetheless, differences between human and pig have been found at the bacteria species level, which are expected to be caused mainly by the environmental factors, nutrition and age [74-76]. The pig breeding under uniform conditions enables to minimize the influence of environment and nutrition on the microbiome.

There are few studies about the link between skin cancer and skin microbiota in skin diseases [77-80]. The first work which studied the relationships between the human skin microbiome and melanoma has shown that the skin microbiome diversity was lower in patients with melanoma than in patients with melanocytic nevi. However, the authors did not detect the association between the cutaneous microbiome and melanoma [81]. Recently, the significant association of the skin microbiome in patients with acral melanoma was investigated at different stages [82]. In our previous study, we showed a significant difference between the healthy skin microbiome and melanoma tissue microbiome using DGGE method [83]. Similarly, in the present study, using high throughput sequencing of the $16 \mathrm{Sr}$ RNA gene, the metagenomic analysis revealed differences in skin microbiome of healthy skin, melanoma surface and melanoma tissue. Alpha diversity showed that the diversity in the healthy skin microbiome was significantly higher than in the melanoma microbiome. Also, the diversity of skin microbiome and faecal microbiome was significantly higher in crossbred white piglets (control animals without tumours) than in MeLiM piglets. The high diversity and richness of host microbiota are generally related to the host health stat [84-86]. Moreover, beta diversity based on Bray Curtis distance showed dissimilarities between microbial communities from multiple sites. The differences between regressive melanoma microbiome and progressive melanoma microbiome were not significant. However, a dynamic shift in the gut and cutaneous microbiome was explored in piglets with melanoma regression following the age, while the microbiota diversity was stable in MeLiM piglets with melanoma progression. That indicates that both the cutaneous microbiome and intestinal microbiome have a strong correlation with the melanoma process by age.

The dominant bacterial phyla in porcine skin microbiota were Firmicutes, Actinobacteria, Proteobacteria and Bacteroidetes in addition to Fusobacteria, which was abundant in the melanoma tissue microbiome of MeLiM piglets. The porcine faecal microbiota was mainly dominated by Firmicutes and Bacteroidetes, besides Fusobacteria, Proteobacteria and Actinobacteria, which were abundant in the faecal microbiota in MeLiM piglets. At the genus level, Lactobacillus, Clostridium, Corynebacterium1, Terrisporobacter and Enterococcus were associated with healthy skin of crossbred piglets whereas the relative abundance of Fusobacterium, Staphylococcus, Trueperella, Streptococcus and Bacteroides were discriminately higher in the melanoma microbiome. Consistent with our previous findings [86], Fusobacterium necrophorum subsp. necrophorum (18,3\%) was the most abundant species in melanoma tissue microbiome of MeLiM piglets besides Staphylococcus hyicus (8,3\%), Trueperella pyogenes (7,1\%) Streptococcus (uncultured bacterium) $(4,3 \%)$ and Staphylococcus chromogenes (2,7\%), while they were considerably low or absents in the healthy skin microbiome. The relative abundance of Lachnospiraceae (9,7\%), Bacteroides (4,2\%), Escherichia-Shigella (2,4\%) and Fusobacterium (1,6\%) (Fusobacterium necrophorum subsp. necrophorum and Fusobacterium gastrosuis) were significantly discriminant bacterial genera in the faecal microbiota of MeLiM piglets, while Prevotella 9 (8,3\%), Prevotellaceae NK3B31 group (4,4\%), Lactobacillus $(5,8 \%)$ and Feacalibacterium $(1,9 \%)$ were the most discriminant bacterial genera in the faecal microbiota of crossbred piglets.

Fusobacterium was associated with pathogenesis in both human and livestock infections [87, 88]. Two subspecies of $F$. necrophorum are recognized. The subsp. necrophorum is more frequently present animal infections, while subsp. funduliforme was isolated from clinical human infections and their virulence is determined by secreting leukotoxin. In humans, $F$. necrophorum is responsible for Lemierre syndrome, which begins as bacterial pharyngitis and rapidly progresses to septic thrombophlebitis of the jugular vein [87-90]. Fusobacterium 
nucleatum was enriched in colorectal carcinoma. It was considered as a risk factor for the progression and severity of pancreatic and colorectal cancers [91, 92]. The active invader species $F$. nucleatum and $F$. periodonticum can independently invade host cells. Fusobacterium nucleatum colonized breast cancer tissue and colorectal cancer tissue, promoted tumour growth and caused cancer progression by inducing immunosuppression using extracellular adhesion and invasion molecule Fusobacterium adhesion (FadA) [93-98]. Gur et al. have demonstrated that the direct interaction of the Fap2 protein of F. nucleatum with the immune cells inhibitory receptor TIGIT (T cell immunoglobulin and ITIM domain) protected melanoma tumours bounded with $F$. nucleatum from NK cell cytotoxicity and T-cell activity [98]. Kalora et al. have identified 11 HLA-bound peptides derived from F. nucleatum, Staphylococcus aureus and Staphylococcus capitis inside melanoma tumour cells that elicited the immune response $[99,100]$.

Staphylococcus hyicus is the major causative agent of piglets' exudative epidermitis [101]. Staphylococcus chromogenes has been identified as a frequent cause of bovine mastitis and intramammary infections $[102,103]$. Interestingly, colonization of mice with Staphylococcus epidermidis, a skin commensal bacteria producing 6-N-hydroxyaminopurine (6-HAP), has reduced the incidence of ultraviolet-induced tumours [10]. Trueperella pyogenes causes diverse diseases in animals like mastitis, liver abscesses and pneumonia and it is rarely a cause of infection in humans [104-106].

Lactobacillus and Corynebacterium species have been shown to produce immunostimulatory metabolites leading to anti-cancer effects. Lactobacillus johnsonii was conducted to an immune-stimulatory effect by producing inosine which is a modulator of response to immune checkpoint blockade therapy and strongly enhanced the antitumor capacities of $\mathrm{T}$ cells in different tumour models including colorectal cancer, bladder cancer, and melanoma, by inducing Th1 differentiation through the inosine-A2AR-cAMP-PKA pathway [107]. A recent study showed that extracellular vesicles derived from Lactobacillus rhamnosus GG showed direct anti-tumour effects on hepatic cancer cell growth [108]. Also, the oral administration of lipoteichoic acid from Lactobacillus rhamnosus decreased the number of UV-induced skin tumours in SKH-1 hairless mice [109] and the administration of Lactobacillus acidophilus may reduce the incidence of colorectal cancer in rat models [110]. The antitumor effect of Corynebacterium parvum has been demonstrated since a very early time [111-113], and it was used as an immunostimulant and antitumor agent. Indeed, the intratumoral injection of Corynebacterium granulosum and Corynebacterium parvum in hamster melanoma showed regression of the tumour and reduction in the number of lung metastases $[114,115]$. Lipton et al. indicated a decreased relapse rate and prolonged survival in patients in stage II, but not in stage I, of malignant melanoma treated with C. parvum when compared with BCG treated patients [116]. Nonetheless, no significant results were observed during the administration of Corynebacterium parvum followed by chemotherapy in patients with metastatic malignant melanoma compared with the group receiving chemotherapy alone [117]. Though, Corynebacterium $s p$ was clinically associated with the progression of acral melanoma [82]. In our results, LDA detected two biomarkers genera from Corynebacteriaceae family: Corynebacterium 1 , which was discriminately abundant in healthy skin microbiome (Fig. S5a, S5b), (Table S3) and Corynebacterium, which was associated with melanoma progression (Fig. 4b), (Table S3).

Among the other bacteria affecting the immune response in cancer, $E$. coli produces colibactin, which may induce adenocarcinomas in human colorectal cancer patients [118]. Bacteroides fragilis led to promote colon tumorigenesis by overstimulating immune responses via $\mathrm{T}$ helper 17 (Th17) cells in mouse colorectal cancer model [119]. Clostridium species may suppress tumour growth in the liver and melanoma by restoring antitumour immunity [120, 121]. Gut microbiome enriched with Faecalibacterium was correlated with increased Immune Checkpoint Inhibitors response and improved immunotherapy response in mouse models and humans with metastatic melanoma $[30,122]$. In a study of metastatic melanoma treated with anti-PD1 immune checkpoint blockade, the patients have reacted differently, responded and non-responded. The diversity of the faecal microbiome of the responders' patients was higher with increased abundance of the Ruminococcaceae and Faecalibacterium, while an increased abundance of Bacteriodales and a much lower bacterial diversity were observed in the non-responders' microbiomes. In animal models, FMT of human gut microbiome enriched in Faecalibacterium to germ-free mice with melanoma showed reduced tumour growth and increased immune response in the tumour microenvironment [30]. The decrease of the relative abundances of opportunistic pathogens in skin microbiota and faecal microbiota of piglets with melanoma regression revealed the maturation of host microbiota following the age when the bacterial composition shifted from dysbiosis to the "healthy" balanced microbiota.

Functional prediction pathways results suggested the potential role of host microbiota in melanoma development. Numerous pieces of evidence have been demonstrated that metabolic disorders involved in carcinogenesis and can be a target to treat cancer $[123,124]$. 
Sporulation and Bacterial motility proteins pathways were significantly higher in melanoma-free skin of piglets (Fig. S8). It was shown that Flagellin, the structural protein subunit of the bacterial flagellum, played a role as an adjuvant, immunomodulator, anti-tumour agent (in melanoma, colon, breast, lungs and prostate cancers) and radioprotective agent [125-127]. Salmonella Typhimurium flagellin stimulates NK cells to produce interferon- $\gamma$ (IFN- $\gamma$ ) [128]. The flagellin genes $(\mathrm{fliC})$ were detected in Clostridium chauvoei, Clostridium haemolyticum, Clostridium novyi types A and B, and Clostridium septicum [129]. Bacterial flagellin enhanced the antitumor response of the activated CD8 $+\mathrm{T}$ cells via TLR5 activation. Consequently, the perforin and granzyme proteins were secreted by activated $\mathrm{CD} 8+\mathrm{T}$ cells and efficiently killed tumour cells [128]. Additionally, a significant reduction in tumour mass was observed after injection of flagellin into human colorectal tumours xenografted into nude mice [130]. Importantly, the vaccination of mice with syngeneic B16-OVA melanoma-derived plasma membrane vesicles engrafted with flagellin-related peptides 9Flg or 42Flg induced a dramatic inhibition of tumour growth and metastasis and resulted in complete tumour regression in lungs [131]. Clostridium novyiNT (non-toxic) is a highly mobile spore-forming organism. Promising antitumour responses in both canine and human clinical studies were described after intratumoral injection of Clostridium novyi-NT spores [132]. Moreover, clostridial spores were considered as an ideal delivery vehicle for anti-cancer agents due to their selective germination in the hypoxic regions of solid tumour, their wide and fast dispersion throughout the tumour and their stability to oxygen and harsh conditions which allowed the immune system to recognize and destroy cancer cells efficiently [133].

Transporters, $\mathrm{ABC}$ transporters, Ribosome, Other ioncoupled transporters_Unclassified, Pyrimidine metabolism, Purine metabolism, Replication, Recombination and repair proteins, Aminoacyl-tRNA biosynthesis, Lipopolysaccharide biosynthesis proteins, Lipopolysaccharide biosynthesis, Bacterial secretion system and Ribosome biogenesis were highly predicted pathways affected by microbiota in MeLiM melanoma tissue (Fig. S8). Recently, ribosome synthesis was designated as a new target in cancer therapy. Moreover, recent research indicated the potential role of ribosomes compositions in tumorigenesis. The increase in ribosome biogenesis was noted in cancer cells which led to an elevation in protein synthesis and unrestrained growth [134]. The production of lipopolysaccharide (LPS) was potentially promoted by Fusobacterium which were abundant in melanoma tissue. LPS from Fusobacterium led to skin inflammation and Shwartzman reaction in rabbits [135]. LPS may promote inflammation via TLR4-mediated NF-kB activation and the production of different inflammatory factors, such as TNF- $\alpha$, IL-6, and IL-1 $\beta$ [136, 137]. Many studies indicated the capacity of LPS to be involved in the progression of various cancers: breast cancer via a 'MyD88BLT2'-linked signalling cascade [138], prostate cancer by activating the NF- $\mathrm{kB}$ pathway [139], gastric cancer through the LPS-NF-kB-PD-L1 axis [140], and oesophageal cancer [141]. A recent study demonstrated the costimulation with Trueperella pyogenes pyolysin and LPSs induced autophagy and ATF6-dependent mechanism in endometrium stromal cells [142].

Amino acid metabolism is linked with tumour progression due to their indispensable role in cancer growth, cancer immunity and the tumour microenvironment $[143,144]$ and therapeutic means for targeting amino acid metabolism were suggested [145]. A potential antitumor effect of a combination of ascorbic acid, lysine, proline, arginine and green tea extract was investigated on human colon cancer cells HCT 116 in vivo (xenograft into male nude mice). Histological studies showed that the mixture supplementation strongly suppressed the growth of tumours by inhibiting Matrix metalloproteinases expression and invasion without toxic effects [146]. Tumour associated myeloid cells have the ability to suppress the protective anti-tumour immune response by targeting arginine metabolism and reducing arginine levels by producing arginases $[147,148]$. In line with these findings result from our previous metabolomic study, where a highly significantly decreased level of arginine in plasma of MeLiM pigs with progression was the most striking difference compared to pigs with spontaneous regression [149]. Up-regulation of proline in melanoma cells compared to melanocytes was observed by de Ingeniis et al. [150]. An inhibition of the gene ALDH18A1 encoding pyrroline-5-carboxylate synthase regulating proline biosynthesis in melanoma cells significantly decreased cultured melanoma cell viability and tumour growth [151, 152].

The predicted functional pathways affected by microbiota were significantly different between regressive melanoma microbiome and progressive melanoma microbiome. It is known that tumour cells accumulate several mutations and changes in metabolic pathways that might lead to cancer proliferation and metastasis. Ribosomes, DNA repair and recombination proteins, Pyrimidine metabolisms, Purine metabolisms were significantly higher in melanoma progression while Bacterial motility proteins. Flagellar assembly, Sporulation and Bacterial chemotaxis were significantly higher in melanoma spontaneous regression (Fig. S9). Consistent with our findings, many studies have assessed the interplay of pyrimidine metabolism in tumorigenesis $[153,154]$. 
Comprehensively, the pyrimidine metabolic rate-limiting enzymes were highly expressed in lung, breast, colon, liver, stomach, and bladder cancer and played a key role in tumour cell proliferation [155]. Pyrimidine analogues acting as antimetabolites are used in cancer treatment for decades [156, 157]. Purines are basic components of nucleotides in cell proliferation, thus impaired purine metabolism is associated with the progression of cancer [158]. Notably, a high amount of purine metabolites has been noticed in tumour cells [159]. It was illustrated that Escherichia coli was able to target lung cancer cells using chemotaxis towards the biochemical factors secreted by carcinoma cells [160]. In faecal microbiota of MeLiM piglets, fatty acids biosynthesis and lipid biosynthesis metabolism were significantly associated with melanoma progression (Fig. S6d). Cancer cells as proliferative active cells require also lipids and fatty acids for cell growth, division, proliferation and survival. Deregulated lipid metabolism is an important metabolic phenotype of cancer cells [161]. Different mechanisms of fatty acids synthesis promoting tumour progression and metastasis were explored $[162,163]$. Upregulations of several phosphatidylcholines were previously observed in MeLiM plasma of pigs with melanoma progression, compared to spontaneous regression [148]. A phospholipid derivative Lysophosphatidic acid (LPA) induces chemotaxis of melanoma cells and LPA degradation by melanoma cells forms a gradient in the tumour microenvironment that drove their spreading [164].

Ribosomes, DNA repair and recombination proteins and Pyrimidine metabolisms pathways, which were associated with melanoma progression in melanoma tissue microbiome, were significantly lower in the faecal microbiota of MeLiM piglets compared to crossbred piglets (Fig. S10) and they were significantly lower in the faecal microbiota of MeLiM piglets with melanoma regression at the age of 8 weeks compared to MeLiM piglets with melanoma regression at the age of 10 and 12 weeks (Fig. S11, S12). However, membrane transport pathways (Transporters, phosphotransferase system (PTS), ABC transporters, and others ion-coupled transporters unclassified) were significantly enriched in melanoma tissue microbiome, in the faecal microbiota of MeLiM piglets compared to crossbred piglets and they were significantly higher in the faecal microbiota of MeLiM piglets with melanoma regression at the age of 8 weeks (Fig. S8, S9, S10, S11, S12). Throughout the age, membrane transport pathways significantly decreased in the melanoma regression group while no significant difference was detected in the melanoma progression group in this pathway.

Alterations of membrane transport pathway were associated with several human diseases including cancer $[141,165,166]$ and caused severe functional influences: increase intestinal permeability, alteration of the balance of substances between both sides of gut mucosa and destruction of the intestinal mucosal barrier. In addition, there is new evidence of the impact of the intestinal microbiome on skin physiology and microbiome [25, 167]. In case of disrupted intestinal barriers, intestinal bacteria and their metabolites have been reported to diffuse in the bloodstream, reach the skin, and disturb skin homeostasis [25, 27]. Based on those observations, our results lead up to deduce that in MeLiM piglets the intestinal permeability was higher and allowed the migration of the intestinal bacteria and its metabolites throughout the blood to gain access to the melanoma microenvironment and enhance tumour growth and proliferation. However, following the age, the intestinal permeability of piglets with melanoma regression was decreased and consequently, the transit of intestinal bacteria and its metabolites from the gut to the skin was restricted which was conducted to melanoma regression.

Depth studies suggested that gut $F$. nucleatum originated from the oral cavity $[168,169]$. Several reports have demonstrated that Fusobacteria can migrate from the oral cavity to other districts of the body via blood circulation or lymphatic circulation systems to cause serious infections in various organs of humans including the head, neck, respiratory tract, brain, colon, liver and lymph nodes to cause metastatic abscess formation [170-175]. Also, recent studies suggested the translocation of Fusobacteria from colorectal cancer cells to metastatic sites attached with the primary tumour cells via Fap2, as part of metastatic tissue colonization [170, 175]. These observations indicate the importance of tumour microbiota as essential components of the tumour microenvironment.

\section{Conclusion}

According to our knowledge, this is the first study exploring the association between gut and skin microbiome changes and melanoma progression. The implementation of MeLiM piglets as an animal model in melanoma progression might be a promising approach. Significant differences were observed in bacterial composition, relative abundances, richness and diversity indexes, and potential functional pathways in skin and gut microbiome of MeLiM piglets with melanoma progression compared to healthy piglets (controls). Fusobacterium and Bacteroides were the common potential biomarkers identified in both gut and skin microbiome in MeLiM piglets with melanoma progression.

The comprehensive analysis of the gut-skin axis is essential to understand the bidirectional cross-talk between the gut and skin tumour microbiome and is regarded as an exciting field of research, with promising 
therapeutic, dermatologic and cosmetic applications. Moreover, the evaluation of the functions of distinct bacteria (biomarker) and their metabolites in host physiology and cancer progression can provide novel insights into the underlying mechanisms and pathways to enhance the efficacy of both anticancer therapy and cancer prevention. Furthermore, a meta-analysis might be potentially expanded to include fungi and viruses in order to fully exploit the interaction network and potential functional between the gut and skin microbiome and melanoma development.

\section{Methods}

\section{Animal experimentation}

All animals used in the study are owned by the Institute of Animal Physiology and Genetics, Czech Academy of Sciences. The study was conducted under the Authorization for the use of experimental animals (No. 71922/2016-MZE-17214) and Authorization for breeding of experimental animals and delivery of experimental animals (No. 9322/2015 MZE-17214) issued by the Ministry of Agriculture of the Czech Republic and approved by the Resort Professional Commission of the Czech Academy of Sciences for Approval of Projects of Experiments on Animals (Projects of Experiments No. 82-2017 and No. 96-2015).

The MeLiM piglets $(n=20)$ and MeLiM x white control pig crossbreds $(n=14)$ were included in the study. The piglets were housed together with sows, fed by the diet appropriate to their age with unlimited access to water and sow milk. According to the phenotype, the piglets were divided into 4 groups: (i) MeLiM piglets with black skin and melanomas undergoing progression $(n=10)$; (ii) MeLiM piglets with black skin and melanomas undergoing spontaneous regression $(n=10)$; (iii) crossbreds with black skin and melanomas undergoing spontaneous regression $(n=4)$; and, (iv) crossbreds with white skin (controls without tumours; $n=10$ ).

The melanoma development (the position, size and shape of tumours, as well as animal body weight) was macroscopically monitored at the $6,8,10$ and 12 weeks of piglet age to assess the progressive or regressive disease development. At the 8,10 , and 12 weeks of age, the scrapings from the melanoma surface and surrounding healthy skin surface $\left(4 \mathrm{~cm}^{2}\right.$ area, approximately $5 \mathrm{~cm}$ from melanoma) were collected using a sterile scalpel blade to a sterile tube. Before the skin surface scraping, the bristles were removed by a different sterile scalpel blade. In these time intervals, the piglet faeces were collected from the rectum into sterile tubes. The scrapings and stool samples were immediately frozen to $-80{ }^{\circ} \mathrm{C}$. In addition, at 8 and 12 weeks of piglet age, the melanomas were collected from animals under Isoflurane and nitric oxide general anaesthesia. After collection, the tumour edges and surface were aseptically removed and the melanoma inner tissue was immediately frozen to liquid nitrogen and stored at $-80{ }^{\circ} \mathrm{C}$ until analysis.

\section{DNA extraction}

The DNA was extracted from stool samples using QIAamp PowerFecal DNA Kit (QIAGEN, Hilden, Germany) and from tissue samples using DNeasy PowerBiofilm Kit (QIAGEN, Hilden, Germany) as per the manufacturer's protocols. The disintegration step was performed with a FastPrep-24 Classic instrument (MP Biologicals) device for $1 \mathrm{~min}$ at a maximum speed of $6.5 \mathrm{~m} / \mathrm{s}$. The elution was done with $60 \mu \mathrm{L}$ of elution buffer. The eluted DNA was stored at $-20{ }^{\circ} \mathrm{C}$ until further use.

\section{Amplification of $16 \mathrm{~S}$ rDNA and purification}

The V4-V5 region of the 16S rRNA gene was amplified to prepare amplicons from the extracted DNA using the primer pair: BactB-F (GGATTAGATACCCTGGTAGT) and BactB-R (CACGACACGAGCTGACG) [176]. mixed with EliZyme HS FAST MIX Red (Elisabeth Pharmacon, Brno, Czech Republic). The PCR conditions were: denaturation for $5 \mathrm{~min}$ at $95{ }^{\circ} \mathrm{C}$, followed by 25 cycles of $30 \mathrm{~s}$ at $95{ }^{\circ} \mathrm{C}, 30 \mathrm{~s}$ at $57{ }^{\circ} \mathrm{C}$ and $30 \mathrm{~s}$ at $72{ }^{\circ} \mathrm{C}$, ending by final elongation for $5 \mathrm{~min}$ at $72{ }^{\circ} \mathrm{C}$. The quality of PCR amplicons was checked by $1.5 \%$ agarose gel electrophoresis $(30 \mathrm{~min}$ at $100 \mathrm{~V})$, then the amplicons were purified using QIAquick PCR Purification Kit (QIAGEN, Hilden, Germany) according to the manufacturer's protocol and the concentration of the purified amplicons was determined by Nanodrop OneC Microvolume UV-Vis Spectrophotometer (Thermo Scientific, Wilmington, USA).

\section{Semi-conductor based Next Generation Sequencing}

For diversity analyses, libraries were prepared from purified amplicons of V4-V5 region of the 16Sr RNA gene (300 bp) by NEBNext ${ }^{\circledR}$ Fast DNA Library Prep Set kit (New England Biolabs, Ipswich, MA, USA). The adaptorligated libraries were purified using AMPure XP beads sizing (Beckman Coulter, Brea, CA, USA). The quality of purified libraries was controlled by High Sensitivity DNA electrophoresis with Agilent 2100 Bioanalyzer instrument (Agilent Technologies, Santa Clara, CA, USA) using the Agilent High Sensitivity DNA Reagents and chips (Agilent Technologies, Santa Clara, CA, USA). The purified libraries were quantified using the KAPA Library Quantification Kit for Ion Torrent Platforms (Roche, Pleasanton, CA, USA) in QuantStudio ${ }^{\mathrm{TM}} 3$ Real-Time PCR System (Thermo Fisher Scientific, Waltham, MA, USA). The pool of equimolar concentration of barcoded libraries was used to prepare a sequencing template with 
Ion Sphere Particles (ISPs) using Ion $\mathrm{PGM}^{\mathrm{TM}} \mathrm{Hi}-\mathrm{Q}^{\mathrm{TM}}$ View OT2 400 Kit (Thermo Fisher Scientific, Waltham, MA, USA) in Ion OneTouchTM 2 instrument. The enrichment of the template positive ISPs was performed on the Ion OneTouchTM ES instrument. The enriched template positive ISPs were loaded in Ion 316TM Chip v2 BC (Thermo Fisher Scientific, Waltham, MA, USA). The sequencing was then performed on an Ion Torrent PGM sequencer (Thermo Fisher Scientific, Waltham, MA, USA) using Ion $\mathrm{PGM}^{\mathrm{TM}} \mathrm{Hi}-\mathrm{Q}^{\mathrm{TM}}$ View Sequencing solutions kit (Thermo Fisher Scientific, Waltham, MA, USA) following the manufacturer's instructions.

\section{Microbiome analysis and statistical analysis}

Bacterial 16S rRNA gene sequences were obtained in FASTQ format and analyzed by QIIME 2 version 2020.2 pipeline [177]. Quality filtering and chimaera excluding were performed using the DADA2 plugin in QIIME2 [178] (via q2-dada2) to extract sequence variants (ASVs). Mafft was used to align the sequences [179] (via q2-alignment) and fasttree was used to construct a phylogenetic tree [180] (via q2-phylogeny). Then, clustering and taxonomy classification was generated using the qiime feature-classifier with VSEARCH based on SILVA database (version 132) with 99\% threshold [181]. The rarefaction was conducted to normalize the data based on reads depth in all samples. Alpha diversity indexes were determined using q2-diversity plugin based on the Kruskal-Wallis test and visualized using the qiime2R (https://github.com/jbisanz/qiime2R) and ggplot2 packages in R-Studio (version 3.6.3) [182-184]. Principal Coordinate Analysis (PCoA) based on Bray Curtis distance diversity metrics (beta diversity) were generated by qiime 2 core-metrics phylogenetic pipeline after rarefaction. The 2-dimensional PCoA plots were generated by qiime2R and ggplot 2 . Non-metric multidimensional scaling (NMDS) plot was performed using phyloseq and ggplot2 and the dissimilarity was based on Bray Curtis distance $[184,185]$. The confidence ellipse represents $95 \%$ of the confidence level. The linear discriminant analysis (LDA) with effect size (LefSe) algorithm [186] in Galaxy module http://hutte nhower.sph.harvard.edu/galaxy/ was used to detect features with significant differential abundance between different biological categories of samples based on the factorial Kruskal-Wallis test and the pairwise Wilcoxon test to identify taxa with significant differential relative abundances at genus level with alpha values of 0.05 and a threshold value of 3.0 on the logarithmic LDA scores for discriminative features $(p<0.05$ and LDA score/ effect-size threshold $=3$ ). Phylogenetic investigation of communities by reconstruction of unobserved states algorithm (PICRUSt v 2.3.0-b) [187] was applied to compare the potential function capacities of the cutaneous microbiome and faecal microbiome among different categories of samples. The functional genes were categorized into KEGG pathways at different subclasses levels 1, 2 and 3 and the resulting abundance table was imported in STAMP v2.1.3 program (Statistical Analysis of Metagenomic Profiles) for statistical analysis [188] by using Non-corrected Welch's t-test type two-sided, with the confidence interval $(\mathrm{CI})$ method of Welch's inverted adjustment of $0.95(p<0.05)$. The relationships among functional capacities were analysed by principal component analysis (PCA).

\section{Abbreviations}

DGGE: Denaturing Gradient Gel Electrophoresis; FASTQ: Fast Adaptive Shrinkage Threshold Algorithm Quality; FMT: Faecal Microbiota Transplantation; KEGG: Kyoto Encyclopedia of Genes and Genomes; LDA: Linear Discriminant Analysis; LPS: Lipopolysaccharide; MeLiM: Melanoma-bearing Libechov Minipig; NMDS: Non-metric Multidimensional Scaling; PCA: Principal Component Analysis; PCoA: Principal Coordinate Analysis; PGM: Personal Genome Machine; PICRUST: Phylogenetic Investigation of Communities by Reconstruction of Unobserved States; STAMP: Statistical Analysis of Metagenomic Profiles.

\section{Supplementary Information}

The online version contains supplementary material available at https://doi. org/10.1186/s12866-022-02458-5.

Additional file 1. Adiagram summarizing the principal steps of the used methods. Drawn by the authors using Adobe Olllustrator $^{\circ} \mathrm{CS6}$.

Additional file 2. Comparison of the alphadiversity of bacterial communities a) in cutaneous microbiome among differentcutaneous samples (healthy skin, melanoma surface and melanoma tissue), b)cutaneous microbiome of different animal breeds (white, crossbred, MeLiM) and c) faecal microbiome of different piglets groups (white control, crossbred withmelanoma regression, MeLiM with melanoma regression and MeLiM with melanomaprogression). Bacterial diversity and richness were estimated by differentalpha diversity indexes: Observed species, Chao, Ace, Shannon, Simpson, InverseSimpson and Fisher index. Kruskal-Wallis pairwise test ( $p$-value $\leq 0.05$ ) wasused to compare between different samples.

Additional file 3. Comparison of the bacterialalpha diversity a) in the cutaneous microbiome of piglets with melanomaregression and piglets with melanoma progression in different cutaneous samples(healthy skin, melanoma surface and melanoma tissue), b) in the cutaneousmicrobiome of piglets with melanoma progression and melanoma regression atdifferent ages $(8,10,12$ weeks) and $c)$ in faecal microbiome of piglets atdifferent stat (control, melanoma progression and melanoma regression) throughout the age $(8,10,12$ weeks). Bacterial diversity and richness wereestimated by different alpha diversity indexes: Observed species, Chao, Ace,Shannon, Simpson, Inverse Simpson and Fisher index. Kruskal-Wallis pairwisetest ( $p$-value $\leq 0.05$ ) was used to compare between different samples.

Additional file 4. Beta diversity of bacterialcommunities using Principal Coordinate Analysis (PCoA) ordinations based on theBray Curtis distance matrix. The dissimilarities between bacterial communitieswere represented by regrouping in distinct clusters: a) in skin microbiomeamong different samples (healthy skin, melanoma surface and melanoma tissue, b)in skin microbiome among multiple samples in different disease conditions(control, melanoma progression and melanoma regression) and c) in faecalmicrobiome of different piglets groups (white control, crossbred with melanomaregression, MeLiM with melanoma regression and MeLiM with melanomaprogression). The confidence level of the ellipse was 95\%. 
Additional file 5. Linear discriminantanalysis (LDA) effect size (LEfSe) at genera level (i) in skin microbiomebetween: a) healthy skin and melanoma surface and b) healthy skin and melanomatissue, and (ii) in faecal microbiome c) between different conditions (healthycontrol, melanoma progression and melanoma regression) and d) between MeLiMpiglets and crossbred animals. Differential abundance between categories wasevaluated based on the factorial Kruskal-Wallis (KW) test and the pairwiseWilcoxon test ( $p<0.05$ and LDA score/effect-size threshold $=3$ ).

\section{Additional file 6 .}

\section{Additional file 7.}

\section{Additional file 8.}

Additional file 9. Functional pathway analysis ofthe cutaneous microbiome and faecal microbiome based on the KEGG database.Extended error bar plot identifying the significant differences in meanproportion (\%) of predicted functional categories a) at second-level KEGGpathway between the healthy skin microbiome and melanoma tissue microbiome, b)at second-level KEGG pathway between melanoma progression and melanomaregression in melanoma tissue microbiome, c) at second-level KEGG pathwaybetween the faecal microbiome of MeLiM piglets and faecal microbiome ofcrossbred piglets and d) at third-level KEGG pathway between the faecalmicrobiome of MeLiM piglets with melanoma progression and MeLiM piglets withmelanoma regression using the STAMP software. The corrected $p$-values that wereshown on the right, were obtained from a Welch's t-test with the confidenceinterval (Cl) method of Welch's inverted adjustment of $0.95(p<0.05)$.

\section{Additional file 10.}

Additional file 11.

\section{Additional file 12.}

Additional file 13. Principal ComponentAnalysis (PCA) of the predicted functional pathways at KEGG level 2 a) betweenthe healthy skin microbiome and melanoma tissue microbiome, b) between melanomaprogression and melanoma regression in melanoma tissue microbiome, c) betweenthe faecal microbiome of MeLiM piglets and faecal microbiome of crossbredpiglets and d) between the faecal microbiome of MeLiM piglets with melanomaprogression and MeLiM piglets with melanoma regression using the STAMP softwarebased on Non-corrected Welch'st-test type two-sided, with the confidenceinterval (Cl)methodofWelch's invertedadjustment of $0.95(p<0.05)$.

\section{Additional file 14.}

\section{Additional file 15.}

\section{Additional file 16.}

Additional file 17. Functional pathway analysis of cutaneous microbiomebased on the KEGG database. Extended error bar plot identifying the significantdifferences in mean proportion (\%) of predicted functional categories at level3 of KEGG pathway between the healthy skin microbiome and melanoma tissue microbiome.

Additional file 18. Functional pathway analysisof cutaneous microbiome based on the KEGG database. Extended error bar plotidentifying the significant differences in mean proportion (\%) of predictedfunctional categories at level 3 of KEGG pathway between melanoma progressionand melanoma regression in melanoma tissue microbiome.

Additional file 19. Functional pathway analysisof faecal microbiome based on the KEGG database. Extended error bar plotidentifying the significant differences in mean proportion (\%) of predictedfunctional categories at level 3 of KEGG pathway between MeLiM piglets andcrossbred piglets.

Additional file 20. Functional pathway analysisof faecal microbiome based on the KEGG database. Extended error bar plotidentifying the significant differences in mean proportion (\%) of predictedfunctional categories at second-level KEGG pathway between MeliM piglets withmelanoma regression at the age of 8 weeks and MeliM piglets with melanomaregression at the age of 10 weeks.

Additional file 21. Functional pathway analysisof faecal microbiome based on the KEGG database. Extended error bar plotidentifying the significant differences in mean proportion (\%) of predictedfunctional categories at second-level KEGG pathway between MeliM piglets withmelanoma regression at the age of 8 weeks and MeliM piglets with melanomaregression at the age of 12 weeks.

Additional file 22. Pairwise comparison ofalpha diversity of cutaneous bacterial communities of animal models (white,crossbred, MeLiM piglets) in different skin samples (healthy skin, melanoma surfaceand melanoma tissue), at different stat (control, melanoma regression andmelanoma regression) at different ages $(8,10,12$ weeks) measured by Chao,Evenness, Shannon and Simpson index of diversity using Kruskal-Wallis pairwisetest ( $p$-value $\leq 0.05$ )

Additional file 23. Pairwise comparison ofalpha diversity of faecal microbiota of animal models (white, crossbred, MeLiMpiglets) at different stat (control, melanoma regression and melanomaregression) at different ages $(8,10,12$ weeks) measured by Chao, Evenness,Shannon and Simpson index of diversity using Kruskal-Wallis pairwise test( $p$-value $\leq 0.05$ ).

Additional file 24. The relative abundances ofthe bacterial community in skin microbiota of several cutaneous samples fromdifferent animal models at different ages at phylum and genus levels.

Additional file $\mathbf{2 5}$. The relative abundances ofthe bacterial community in the faecal microbiota of different animal models atdifferent ages at phylum and genus levels.

Additional file 26. Predicted functional KEGGpathways at levels 1,2 and 3 in the skin microbiome of several cutaneoussamples (healthy skin, melanoma surface and melanoma tissue) from differentanimal models (white, crossbred, MeLiM piglets) at different disease states(control, melanoma regression and melanoma regression).

Additional file 27. Predicted functional KEGGpathways at levels 1,2 and 3 in the faecal microbiota of different animalmodels (white, crossbred, MeLiM piglets) at different disease stat (control,melanoma regression and melanoma regression)

\section{Acknowledgements}

We would like to thank Jitka Klucinova and Jaroslava Sestakova for their excellent technical assistance. The authors are also grateful for the human resources support by the "PPLZ" programme of CAS.

\section{Authors' contributions}

All authors contributed significantly to the manuscript. Study design: CM, HKS, JM; methodology: CM, HKS, JC, VH, JM; animal handling and monitoring: HKS, JC, VC, AP, VH; sampling: HKS, JC, VC, AP, VH; NGS experiment: CM, JM; statistical analyses: CM, JM; writing — original draft preparation: CM, HKS; writingreview and editing: all authors; supervision: HKS, JM; project administration: CM, HKS, JM; funding acquisition: HKS, JM. All authors have read and agreed to the published version of the manuscript.

\section{Funding}

This study was supported by the Ministry of education, youth and sports from the Operational Programme Research, Development and Education projects Center for Tumor Ecology-Research of the Cancer Microenvironment Supporting Cancer Growth and Spread (reg. No. CZ.02.1.01/0.0/0.0/16_019/0000785), that provided sources for animal experiments and sample collection, and the Project Excellence (No. CZ.02.1.01/0.0/0.0/15_003/0000460), that provided sources for molecular biology analyses. The funding bodies played no role in the design of the study and collection, analysis, and interpretation of data and in writing the manuscript.

\section{Availability of data and materials}

The datasets used and/or analyzed during the current study are available from the corresponding author on reasonable request.

\section{Declarations}

\section{Ethics approval and consent to participate}

This study was approved by the Resort Professional Commission of the Czech Academy of Sciences for Approval of Projects of Experiments on Animals (Projects of Experiments No. 82-2017 and No. 96-2015). 


\section{Consent for publication \\ Not applicable}

\section{Competing interests}

The authors declare no competing interests.

\begin{abstract}
Author details
${ }^{1}$ Laboratory of Anaerobic Microbiology, Institute of Animal Physiology and Genetics of the Czech Academy of Sciences, Videnska 1083, 142 20 Prague, Czech Republic. ${ }^{2}$ Laboratory of Applied Proteome Analyses, Institute of Animal Physiology and Genetics of the Czech Academy of Sciences, Rumburska 89, 27721 Libechov, Czech Republic. ${ }^{3}$ Department of Radiobiology, Faculty of Military Health Sciences, University of Defence, Trebesska 1575, 50001 Hradec Kralove, Czech Republic. ${ }^{4}$ Department of Cell Biology, Faculty of Science, Charles University, Vinicna 7, 12800 Prague, Czech Republic.
\end{abstract}

\section{Received: 9 November 2021 Accepted: 29 January 2022}

Published online: 25 February 2022

\section{References}

1. Cummins DL, Cummins JM, Pantle H, Silverman MA, Leonard AL, Chanmugam A. Cutaneous malignant melanoma. Mayo Clin Proc. 2006;81(4):500-7. https://doi.org/10.4065/81.4.500.

2. da Costa NF, Fernandes NC, Borges MRMM. Study of the histopathological types of cutaneous melanoma in palmas-TO from 2001 to 2011. An Bras Dermatol. 2015;90(5):638-45. https://doi.org/10.1590/abd18064841.20153528

3. Gershenwald JE, Guy GP. Stemming the rising incidence of melanoma: calling prevention to action. J Natl Cancer Inst. 2016;108(1):2015-7. https://doi.org/10.1093/jnci/djv381.

4. Carr S, Smith C, Wernberg J. Epidemiology and risk factors of melanoma. Surg Clin North Am. 2020;100(1):1-12. https://doi.org/10.1016/j. suc.2019.09.005

5. Conforti C, Zalaudek I. Epidemiology and risk factors of melanoma: a review. Dermatol Pract Concept. 2021;11:2021161S. https://doi.org/10. 5826/dpc.11s1a161s.

6. Belbasis L, Stefanaki I, Stratigos AJ, Evangelou E. Non-genetic risk factors for cutaneous melanoma and keratinocyte skin cancers: an umbrella review of meta-analyses. J Dermatol Sci. 2016;84(3):330-9. https://doi. org/10.1016/j.jdermsci.2016.09.003.

7. Kovács T, Mikó E, Ujlaki G, Sári Z, Bai P. The microbiome as a component of the tumor microenvironment. Adv Exp Med Biol. 2020;1225:137-53. https://doi.org/10.1007/978-3-030-35727-6_10.

8. Zhao K, Hu Y. Microbiome harbored within tumors: a new chance to revisit our understanding of cancer pathogenesis and treatment. Signal Transduct Target Ther. 2020;5(1):2-4. https://doi.org/10.1038/ s41392-020-00244-1.

9. Xavier JB, Young VB, Skufca J, et al. The cancer microbiome: distinguishing direct and indirect effects requires a systemic view. Trends in Cancer. 2020;6(3):192-204. https://doi.org/10.1016/j.trecan.2020. 01.004 .

10. Nakatsuji T, Chen TH, Butcher AM, et al. A commensal strain of Staphylococcus epidermidis protects against skin neoplasia. Sci Adv. 2018;4(2):eaao4502. https://doi.org/10.1126/sciadv.aao4502.

11. Byrd AL, Belkaid Y, Segre JA. The human skin microbiome. Nat Rev Microbiol. 2018;16(3):143-55. https://doi.org/10.1038/nrmicro.2017.157.

12. Chen YE, Tsao H. The skin microbiome: current perspectives and future challenges. J Am Acad Dermatol. 2013;69(1):143-155.e3. https://doi.org/ 10.1016/j.jaad.2013.01.016.

13. Stacy A, Belkaid Y. Microbial guardians of skin health. Science. 2019;363(6424):227-8. https://doi.org/10.1126/science.aat4326.

14. Belkaid $Y$, Hand TW. Role of the microbiota in immunity and inflammation. Cell. 2014;157(1):121-41. https://doi.org/10.1016/j.cell.2014.03.011.

15. Schommer NN, Gallo RL. Structure and function of the human skin microbiome. Trends Microbiol. 2013;21(12):660-8. https://doi.org/10. 1016/j.tim.2013.10.001.

16. Ansaldo E, Farley TK, Belkaid Y. Control of immunity by the microbiota. Annu Rev Immunol. 2021;39:449-79. https://doi.org/10.1146/ annurev-immunol-093019-112348.
17 Fyhrquist N, Salava A, Auvinen P, Lauerma A. Skin biomes. Curr Allergy Asthma Rep. 2016;16(5):40. https://doi.org/10.1007/ s11882-016-0618-5.

18. Zhou H, Shi L, Ren Y, Tan X, Liu W, Liu Z. Applications of human skin microbiota in the cutaneous disorders for ecology-based therapy. Front Cell Infect Microbiol. 2020;10:1-12. https://doi.org/10.3389/fcimb.2020.570261.

19. Erin Chen Y, Fischbach MA, Belkaid Y. Skin microbiota-host interactions. Nature. 2018;553(7689):427-36. https://doi.org/10.1038/nature25177.

20. Coggshall K, Brooks L, Nagarajan P, Arron ST. The microbiome and its contribution to skin cancer. Curr Cancer Res. 2019:87-106. https://doi. org/10.1007/978-3-030-04155-7_5

21. Sacksteder MR. Brief communication: occurrence of spontaneous tumors in the germfree F344 Rat. J Natl Cancer Inst. 1976;57(6):1371-3. https://doi.org/10.1093/jnci/57.6.1371.

22. Mittal D, Saccheri F, Vénéreau E, Pusterla T, Bianchi ME, Rescigno M. TLR4-mediated skin carcinogenesis is dependent on immune and radioresistant cells. EMBO J. 2010;29(13):2242-52. https://doi.org/10. 1038/emboj.2010.94.

23. Sherwani MA, Tufail S, Muzaffar AF, Yusuf N. The skin microbiome and immune system: potential target for chemoprevention? Photodermatol Photoimmunol Photomed. 2018;34(1):25-34. https://doi.org/10.1111/ phpp.12334.

24. Schwabe RF, Jobin C. The microbiome and cancer. Nat Rev Cancer. 2013;13(11):800-12. https://doi.org/10.1038/nrc3610.

25. O'Neill CA, Monteleone G, McLaughlin JT, Paus R. The gut-skin axis in health and disease: a paradigm with therapeutic implications. BioEssays. 2016;38(11):1167-76. https://doi.org/10.1002/bies.201600008.

26. De PB, Grine L, Debaere M, Maes A, Paetzold B, Callewaert C. Gut-skin axis: current knowledge of the interrelationship between microbial dysbiosis and skin conditions. Microorganisms. 2021;9(2):1-33. https:// doi.org/10.3390/microorganisms9020353.

27. Salem I, Ramser A, Isham N, Ghannoum MA. The gut microbiome as a major regulator of the gut-skin axis. Front Microbiol. 2018;9(JUL):1-14. https://doi.org/10.3389/fmicb.2018.01459.

28. Frankel AE, Coughlin LA, Kim J, et al. Metagenomic shotgun sequencing and unbiased metabolomic profiling identify specific human gut microbiota and metabolites associated with immune checkpoint therapy efficacy in melanoma patients. Neoplasia (United States). 2017;19(10):848-55. https://doi.org/10.1016/j.neo.2017.08.004.

29. Davar D, Dzutsev AK, McCulloch JA, et al. Fecal microbiota transplant overcomes resistance to anti-PD-1 therapy in melanoma patients. Science. 2021;371(6529):595-602. https://doi.org/10.1126/science. abf3363.

30. Gopalakrishnan V, Spencer CN, Nezi L, et al. Gut microbiome modulates response to anti-PD-1 immunotherapy in melanoma patients. Science. 2018;359(6371):97-103. https://doi.org/10.1126/science.aan4236.

31. Dai Z, Zhang J, Wu Q, et al. Intestinal microbiota: a new force in cancer immunotherapy. Cell Commun Signal. 2020;18(1):1-16. https://doi.org/ 10.1186/s12964-020-00599-6.

32 Matson V, Fessler J, Bao R, et al. The commensal microbiome is associated with anti-PD-1 efficacy in metastatic melanoma patients. Science. 2018;359(6371):104-8. https://doi.org/10.1126/science.aao3290.

33 Ansaldo BE, Belkaid Y. How microbiota improve immunotherapy. Science. 2021;373:966-7. https://doi.org/10.1126/science.abl3656.

34. Baruch EN, Youngster I, Ben-betzalel G, Ortenberg R, Lahat A, Katz L. Fecal microbiota transplant promotes response in immunotherapyrefractory melanoma patients. Science. 2020;5920(December):1-16.

35. Limeta A, Ji B, Levin M, Gatto F, Nielsen J. Meta-analysis of the gut microbiota in predicting response to cancer immunotherapy in metastatic melanoma. JCI Insight. 2020;5(23):e140940. https://doi.org/10. 1172/jci.insight.140940.

36. Warner AB, McQuade JL. Modifiable Host Factors in Melanoma: Emerging Evidence for Obesity, Diet, Exercise, and the Microbiome. Curr Oncol Rep. 2019;21(8):72. https://doi.org/10.1007/s11912-019-0814-2.

37. Shaikh FY, Gills JJ, Sears CL. Impact of the microbiome on checkpoint inhibitor treatment in patients with non-small cell lung cancer and melanoma. EBioMedicine. 2019;48:642-7. https://doi.org/10.1016/j. ebiom.2019.08.076

38. Li Y, Tinoco R, Elmén L, et al. Gut microbiota dependent anti-tumor immunity restricts melanoma growth in Rnf5 -/- mice. Nat Commun. 2019;10(1):1492. https://doi.org/10.1038/s41467-019-09525-y. 
39. Wang M, Donovan SM. Human microbiota-associated swine: current progress and future opportunities. ILAR J. 2015;56(1):63-73. https://doi. org/10.1093/ilar/ilv006.

40 Guilloteau P, Zabielski R, Hammon HM, Metges CC. Nutritional programming of gastrointestinal tract development. Is the pig a good model for man? Nutr Res Rev. 2010;23(1):4-22. https://doi.org/10.1017/S0954 422410000077.

41. Meurens F, Summerfield A, Nauwynck H, Saif L, Gerdts V. The pig: a model for human infectious diseases. Trends Microbiol. 2012;20(1):50-7. https://doi.org/10.1016/j.tim.2011.11.002.

42. Bourneuf E, Du ZQ, Estellé J, et al. Genetic and functional evaluation of MITF as a candidate gene for cutaneous melanoma predisposition in pigs. Mamm Genome. 2011;22(9-10):602-12. https://doi.org/10.1007/ s00335-011-9334-6.

43. Horak V, Palanova A, Cizkova J, Miltrova V, Vodicka P, Skalnikova HK. Melanoma-bearing libechov minipig (MeLiM): the unique swine model of hereditary metastatic melanoma. Genes (Basel). 2019;10(11):915. https://doi.org/10.3390/genes10110915.

44. Planska D, Burocziova M, Strnadel J, Horak V. Immunohistochemical analysis of collagen IV and laminin expression in spontaneous melanoma regression in the melanoma-bearing libechov minipig. Acta Histochem Cytochem. 2015;48(1):15-26. https://doi.org/10.1267/ahc.14020.

45. Guran R, Vanickova L, Horak V, et al. MALDI MSI of MeLiM melanoma: searching for differences in protein profiles. PLoS One. 2017;12(12): e0189305. https://doi.org/10.1371/journal.pone.0189305.

46. Planska D, Kovalska J, Cizkova J, Horak V. Tissue rebuilding during spontaneous regression of melanoma in the melanoma-bearing libechov minipig. Anticancer Res. 2018;38(8):4629-36. https://doi.org/10.21873/ anticanres.12767.

47. Fortyn K, et al. Exceptional occurrence and extent of malignant melanoma in pig. Veterinární medicína. 1998;43(3):97-91.

48. Tilg $H$, Adolph TE, Gerner RR, Moschen AR. The intestinal microbiota in colorectal cancer. Cancer Cell. 2018;33(6):954-64. https://doi.org/10. 1016/j.ccell.2018.03.004

49. Cheng Y, Ling Z, Li L. The intestinal microbiota and colorectal cancer. Front Immunol. 2020;11:1-13. https://doi.org/10.3389/fimmu.2020. 615056.

50. Gagnière J, Raisch J, Veziant J, et al. Gut microbiota imbalance and colorectal cancer. World J Gastroenterol. 2016;22(2):501-18. https://doi. org/10.3748/wjg.v22.i2.501.

51. Janney A, Powrie F, Mann EH. Host-microbiota maladaptation in colorectal cancer. Nature. 2020;585(7826):509-17. https://doi.org/10.1038/ s41586-020-2729-3.

52. Meng C, Bai C, Brown TD, Hood LE, Tian Q. Human gut microbiota and gastrointestinal cancer. Genomics, Proteomics Bioinforma. 2018;16(1):33-49. https://doi.org/10.1016/j.gpb.2017.06.002.

53. Engstrand L, Graham DY. Microbiome and gastric cancer. Dig Dis Sci. 2020;65(3):865-73. https://doi.org/10.1007/s10620-020-06101-z

54. Yu LX, Schwabe RF. The gut microbiome and liver cancer: mechanisms and clinical translation. Nat Rev Gastroenterol Hepatol. 2017;14(9):52739. https://doi.org/10.1038/nrgastro.2017.72.

55. McAllister F, Khan MAW, Helmink B, Wargo JA. The tumor microbiome in pancreatic cancer: bacteria and beyond. Cancer Cell. 2019;36(6):577-9. https://doi.org/10.1016/j.ccell.2019.11.004.

56. Li Q, Jin M, Liu Y, Jin L. Gut microbiota: its potential roles in pancreatic cancer. Front Cell Infect Microbiol. 2020;10(10):1-9. https://doi.org/10. 3389/fcimb.2020.572492.

57. Zheng $Y$, Fang Z, Xue Y, et al. Specific gut microbiome signature predicts the early-stage lung cancer. Gut Microbes. 2020;11(4):1030-42. https://doi.org/10.1080/19490976.2020.1737487.

58. Liu X, Cheng Y, Zang D, et al. The role of gut microbiota in lung cancer: from carcinogenesis to immunotherapy. Front Oncol. 2021;11(August):1-13. https://doi.org/10.3389/fonc.2021.720842.

59. Zhou H, Liu J, Shen J, Fang W, Zhang L. Gut microbiota and lung cancer: a mendelian randomization study. JTO Clin Res Reports. 2020;1(3): 100042. https://doi.org/10.1016/j.jtocrr.2020.100042.

60. Ingman WV. The gut microbiome: a new player in breast cancer metastasis. Cancer Res. 2019;79(14):3539-41. https://doi.org/10.1158/ 0008-5472.CAN-19-1698.
61. Zhang J, Xia Y, Sun J. Breast and gut microbiome in health and cancer. Genes Dis. 2021;8(5):581-9. https://doi.org/10.1016/j.gendis.2020.08. 002.

62. Bajic P, Wolfe AJ, Gupta GN. The urinary microbiome: implications in bladder cancer pathogenesis and therapeutics. Urology. 2019;126:10-5. https://doi.org/10.1016/j.urology.2018.12.034.

63. He C, Li B, Huang L, et al. Gut microbial composition changes in bladder cancer patients: a case-control study in Harbin China. Asia Pac J Clin Nutr. 2020;29(2):395-403. https://doi.org/10.6133/apjcn.202007_29(2). 0022.

64. Andreeva NV, Gabbasova RR, Grivennikov SI. Microbiome in cancer progression and therapy. Curr Opin Microbiol. 2020;56:118-26. https:// doi.org/10.1016/j.mib.2020.09.001.

65. Cheng WY, Wu CY, Yu J. The role of gut microbiota in cancer treatment: friend or foe? Gut. 2020;69(10):1867-76. https://doi.org/10.1136/ gutjnl-2020-321153.

66. Lopez LR, Bleich RM, Arthur JC. Microbiota effects on carcinogenesis: initiation, promotion, and progression. Annu Rev Med. 2021;72:243-61. https://doi.org/10.1146/annurev-med-080719-091604.

67. Raza MH, Gul K, Arshad A, et al. Microbiota in cancer development and treatment. J Cancer Res Clin Oncol. 2019;145(1):49-63. https://doi.org/ 10.1007/s00432-018-2816-0.

68 Belkaid Y, Segre JA. Dialogue between skin microbiota and immunity. Science. 2014;346(6212):954-9. https://doi.org/10.1126/science.12601 44.

69. Skowron K, Bauza-kaszewska J, Kraszewska Z, et al. Human skin microbiome: impact of intrinsic and extrinsic factors on skin microbiota. Microorganisms. 2021;9(3):1-20. https://doi.org/10.3390/microorgan isms9030543.

70 lida N, Dzutsev A, Stewart CA, et al. Commensal bacteria control cancer response to therapy by modulating the tumor microenvironment. Science. 2013;342(6161):967-70. https://doi.org/10.1126/science.1240527.

71. Belkaid Y, Tamoutounour S. The influence of skin microorganisms on cutaneous immunity. Nat Rev Immunol. 2016;16(6):353-66. https://doi. org/10.1038/nri.2016.48.

72. McQuade JL, Ologun GO, Arora R, Wargo JA. Gut Microbiome Modulation Via Fecal Microbiota Transplant to Augment Immunotherapy in Patients with Melanoma or Other Cancers. Curr Oncol Rep. 2020;22(7):74. https://doi.org/10.1007/s11912-020-00913-y.

73. Baruch EN, Youngster I, Ben-Betzalel G, et al. Fecal microbiota transplant promotes response in immunotherapy-refractory melanoma patients. Science. 2021;371(6529):602-9. https://doi.org/10.1126/science.abb59 20

74. Heinritz SN, Mosenthin R, Weiss E. Use of pigs as a potential model for research into dietary modulation of the human gut microbiota. Nutr Res Rev. 2013;26(2):191-209. https://doi.org/10.1017/S09544224130001 52.

75. Gonzalez LM, Moeser AJ, Blikslager AT. Porcine models of digestive disease: the future of large animal translational research. Transl Res. 2015;166(1):12-27. https://doi.org/10.1016/j.trsl.2015.01.004.

76. McIntyre MK, Peacock TJ, Akers KS, Burmeister DM. Initial characterization of the pig skin bacteriome and its effect on in vitro models of wound healing. PLoS One. 2016;11(11): e0166176. https://doi.org/10. 1371/journal.pone.0166176.

77. Coates M, Lee MJ, Norton D, MacLeod AS. The skin and intestinal microbiota and their specific innate immune systems. Front Immunol. 2019;10:1-11. https://doi.org/10.3389/fimmu.2019.02950.

78. Yu Y, Champer J, Beynet D, Kim J, Friedman AJ. The role of the cutaneous microbiome in skin cancer: lessons learned from the gut. J Drugs Dermatol. 2015;14(5):461-5. https://doi.org/10.1016/j.jaad.2015.02.628.

79. Ellis SR, Nguyen $M$, Vaughn AR, et al. The skin and gut microbiome and its role in common dermatologic conditions. Microorganisms. 2019;7(11):1-19. https://doi.org/10.3390/microorganisms7110550.

80. Shinno-Hashimoto $\mathrm{H}$, Hashimoto $\mathrm{Y}$, Wei $\mathrm{Y}$, et al. Abnormal composition of microbiota in the gut and skin of imiquimod-treated mice. Sci Rep. 2021;11(1):1-11. https://doi.org/10.1038/s41598-021-90480-4.

81. Salava A, Aho V, Pereira P, et al. Skin microbiome in melanomas and melanocytic nevi. Eur J Dermatology. 2016;26(1):49-55. https://doi.org/ 10.1684/ejd.2015.2696. 
82. Mizuhashi S, Kajihara I, Sawamura S, et al. Skin microbiome in acra melanoma: corynebacterium is associated with advanced melanoma. J Dermatol. 2021;48(1):e15-6. https://doi.org/10.1111/1346-8138.15633.

83. Mrázek J, Mekadim C, Kučerová P, et al. Melanoma-related changes in skin microbiome. Folia Microbiol. 2019;64(3):435-42. https://doi.org/10. 1007/s12223-018-00670-3.

84. Mosca A, Leclerc M, Hugot JP. Gut microbiota diversity and human diseases: should we reintroduce key predators in our ecosystem? Front Microbiol. 2016;7(MAR):1-12. https://doi.org/10.3389/fmicb. 2016.00455

85. Lozupone CA, Stombaugh Jl, Gordon Jl, Jansson JK, Knight R. Diversity, stability and resilience of the human gut microbiota. Nature. 2012;489(7415):220-30. https://doi.org/10.1038/nature11550.

86. Le Chatelier E, Nielsen T, Qin J, et al. Richness of human gut microbiome correlates with metabolic markers. Nature. 2013;500(7464):541-6. https://doi.org/10.1038/nature12506.

87. Nagaraja TG, Narayanan SK, Stewart GC, Chengappa MM. Fusobacterium necrophorum infections in animals: pathogenesis and pathogenic mechanisms. Anaerobe. 2005;11(4):239-46. https://doi.org/10.1016/j. anaerobe.2005.01.007.

88. Riordan T. Human infection with Fusobacterium necrophorum (Necrobacillosis), with a focus on Lemierre's syndrome. Clin Microbiol Rev. 2007;20(4):622-59. https://doi.org/10.1128/CMR.00011-07.

89. Kumar A, Anderson D, Amachawadi RG, Nagaraja TG, Narayanan SK. Characterization of Fusobacterium necrophorum isolated from llama and alpaca. J Vet Diagnostic Investig. 2013;25(4):502-7. https://doi.org/ 10.1177/1040638713491407.

90. Narayanan SK, Nagaraja TG, Chengappa MM, Stewart GC. Cloning, sequencing, and expression of the leukotoxin gene from Fusobacterium necrophorum. Infect Immun. 2001;69(9):5447-55. https://doi.org/ 10.1128/IAl.69.9.5447-5455.2001.

91. Kostic AD, Gevers D, Pedamallu CS, et al. Genomic analysis identifies association of Fusobacterium with colorectal carcinoma. Genome Res. 2012;22(2):292-8. https://doi.org/10.1101/gr.126573.111.

92. Kelly D, Yang L, Pei Z. Gut microbiota, Fusobacteria, and colorectal cancer. Diseases. 2018;6(4):109. https://doi.org/10.3390/diseases6040109.

93. Abed J, Maalouf N, Parhi L, Chaushu S, Mandelboim O, Bachrach G. Tumor targeting by Fusobacterium nucleatum: a pilot study and future perspectives. Front Cell Infect Microbiol. 2017;7(JUN):1-5. https://doi. org/10.3389/fcimb.2017.00295.

94. Kaplan CW, Lux R, Huynh T, Jewett A, Shi W, Haake SK. Fusobacterium nucleatum apoptosis-inducing outer membrane protein. J Dent Res. 2005;84(8):700-4. https://doi.org/10.1177/154405910508400803.

95. Hamada T, Zhang X, Mima K, et al. Fusobacterium nucleatum in colorectal cancer relates to immune response differentially by tumor microsatellite instability status. Cancer Immunol Res. 2018;6(11):132736. https://doi.org/10.1158/2326-6066.CIR-18-0174.

96. Mima K, Nishihara R, Qian ZR, et al. Fusobacterium nucleatum in colorectal carcinoma tissue and patient prognosis. Gut. 2016;65(12):197380. https://doi.org/10.1136/gutjnl-2015-310101.

97. Parhi L, Alon-Maimon T, Sol A, et al. Breast cancer colonization by Fusobacterium nucleatum accelerates tumor growth and metastatic progression. Nat Commun. 2020;11(1):1-12. https://doi.org/10.1038/ s41467-020-16967-2.

98. Gur C, Ibrahim Y, Isaacson B, et al. Binding of the Fap2 protein of fusobacterium nucleatum to human inhibitory receptor TIGIT protects tumors from immune cell attack. Immunity. 2015;42(2):344-55. https:// doi.org/10.1016/j.immuni.2015.01.010

99. Kalaora S, Nagler A, Nejman D, et al. Identification of bacteria-derived HLA-bound peptides in melanoma. Nature. 2021:592(7852):138-43. https://doi.org/10.1038/s41586-021-03368-8

100. Jain T, Sharma P, Are AC, Vickers SM, Dudeja V. New insights into the cancer-microbiome-immune axis: decrypting a decade of discoveries. Front Immunol. 2021;12(February):622064. https://doi.org/10.3389/ fimmu.2021.622064.

101. Sato H, Tanabe T, Nakanowatari M, et al. Isolation of Staphylococcus hyicus subsp. hyicus from pigs affected with exudative epidermitis and experimental infection of piglets with isolates. Kitasato Arch Exp Med. 1990:63(2-3):119-30 PMID: 2096257

102. Andresen LO, Ahrens P, Daugaard L, Bille-Hansen V. Exudative epidermitis in pigs caused by toxigenic Staphylococcus chromogenes. Vet
Microbiol. 2005;105(3-4):291-300. https://doi.org/10.1016/j.vetmic. 2004.12.006.

103. Huebner R, Mugabi R, Hetesy G, et al. Characterization of genetic diversity and population structure within staphylococcus chromogenes by multilocus sequence typing. PLoS One. 2021;16(3 March):1-17. https:// doi.org/10.1371/journal.pone.0243688.

104. Azamgarhi T, Warren S. A case of Trueperella pyogene causing prosthetic joint infection. J Bone Jt Infect. 2020;6(3):47-50. https://doi.org/ 10.5194/jbji-6-47-2020.

105. Ribeiro MG, Risseti RM, Bolaños CAD, et al. Trueperella pyogenes multispecies infections in domestic animals: a retrospective study of 144 cases (2002 to 2012). Vet Q. 2015;35(2):82-7. https://doi.org/10.1080/ 01652176.2015 .1022667$.

106. Rzewuska M, Kwiecień E, Chrobak-Chmiel D, Kizerwetter-Świda M, Stefańska l, Gieryńska M. Pathogenicity and virulence of trueperella pyogenes: a review. Int J Mol Sci. 2019;20(11):2737. https://doi.org/10. 3390/ijms20112737.

107 Mager LF, Burkhard R, Pett N, et al. Microbiome-derived inosine modulates response to checkpoint inhibitor immunotherapy. Science. 2020;369(6509):1481-9. https://doi.org/10.1126/SCIENCE.ABC3421.

108. Behzadi E, Mahmoodzadeh Hosseini H, Imani Fooladi AA. The inhibitory impacts of Lactobacillus rhamnosus GG-derived extracellular vesicles on the growth of hepatic cancer cells. Microb Pathog. 2017;110:1-6. https://doi.org/10.1016/j.micpath.2017.06.016.

109. Friedrich $A D$, Campo VE, Cela EM, et al. Oral administration of lipoteichoic acid from Lactobacillus rhamnosus GG overcomes UVBinduced immunosuppression and impairs skin tumor growth in mice. Eur J Immunol. 2019;49(11):2095-102. https://doi.org/10.1002/eji.20184 8024.

110. Mclntosh GH, Royle PJ, Playne MJ. A probiotic strain of L. acidophilus reduces $\mathrm{DMH}$-induced large intestinal tumors in male sprague-dawley rats. Nutr Cancer. 1999;35(2):153-9. https://doi.org/10.1207/S1532 7914 NC352 9.

111. Halpern B, Biozzi G, Stiffel C, et al. Inhibition of tumour growth by administration of killed Corynebacterium parvum. Nature. 1966:212:853-4. https://doi.org/10.1038/212853a0.

112. Woodruff MF, Boak JL. Inhibitory effect of injection of Corynebacterium parvum on the growth of tumour transplants in isogenic hosts. $\mathrm{Br} J$ Cancer. 1966;20(2):345-55. https://doi.org/10.1038/bjc.1966.42.

113 Baum M, Breese M. Antitumour effect of Corynebacterium parvum. Possible mode of action. Br J Cancer. 1976;33(4):468-73. https://doi.org/ 10.1038/bjc.1976.73.

114. Dimitrov N V., Chouroulinkov I, Israel L, O'Rangers JJ. Regression of hamster melanoma by Corynebacterium parvum. Corynebacterium parvum. 1975:276-283. doi:https://doi.org/10.1007/978-1-4613-4428-5_31

115. Paslin D, Dimitrov NV, Heaton C. Regression of a transplantable hamster melanoma by intralesional injections of Corynebacterium granulosum. J Natl Cancer Inst. 1974;52(2):571-3. https://doi.org/10. 1093/jnci/52.2.571.

116. Lipton A, Harvey HA, Lawrence B, et al. Corynebacterium parvum versus BCG adjuvant immunotherapy in human malignant melanoma. Cancer. 1983;51(1):57-60. https://doi.org/10.1002/1097-0142(19830101)51:1\% 3c57::aid-cncr2820510114\%3e3.0.co:2-v.

117. Thatcher N, Wagstaff J, Mene A, Smith D, Orton C, Craig P. Corynebacterium parvum followed by chemotherapy (actinomycin D and DTIC) compared with chemotherapy alone for metastatic malignant melanoma. Eur J Cancer Clin Oncol. 1986;22(8):1009-14. https://doi.org/10. 1016/0277-5379(86)90069-6.

118. Arthur JC, Perez-chanona E, Mühlbauer M, et al. NIH Public Access. 2013;338(6103):120-3. https://doi.org/10.1126/science.1224820.Intes tinal.

119. Wu S, Rhee KJ, Albesiano E, et al. A human colonic commensal promotes colon tumorigenesis via activation of T helper type $17 \mathrm{~T}$ cell responses. Nat Med. 2009;15(9):1016-22. https://doi.org/10.1038/ nm.2015.

120. Ma C, Han M, Heinrich B, et al. Gut microbiome-mediated bile acid metabolism regulates liver cancer via NKT cells. Science. 2018;360(6391):eaan5931. https://doi.org/10.1126/science.aan5931.

121. Van Mellaert L, Wei MQ, Anné J. Live Clostridia: A Powerful Tool in Tumor Biotherapy. Emerg Cancer Ther Microb Approaches Biotechnol Tools. 2010:71-97. doi:https://doi.org/10.1002/9780470626528.ch4 
122. Chaput N, Lepage $P$, Coutzac C, et al. Baseline gut microbiota predicts clinical response and colitis in metastatic melanoma patients treated with ipilimumab. Ann Oncol. 2017;28(6):1368-79. https://doi.org/10. 1093/annonc/mdx108.

123. Tsvetikova SA, Koshel El. Microbiota and cancer: host cellular mechanisms activated by gut microbial metabolites. Int J Med Microbiol. 2020;310(4): 151425. https://doi.org/10.1016/j.ijmm.2020.151425.

124. Luengo A, Gui DY, Vander Heiden MG. Targeting metabolism for cancer therapy. Cell Chem Biol. 2017;24(9):1161-80. https://doi.org/10.1016/j. chembiol.2017.08.028.

125. Hajam IA, Dar PA, Shahnawaz I, Jaume JC, Lee JH. Bacterial flagellin-a potent immunomodulatory agent. Exp Mol Med. 2017;49(9):1-15. https://doi.org/10.1038/emm.2017.172.

126. Sfondrini L, Rossini A, Besusso D, et al. Antitumor activity of the TLR-5 ligand flagellin in mouse models of cancer. J Immunol. 2006;176(11):6624-30. https://doi.org/10.4049/jimmunol.176.11.6624.

127. De Melo FM, Braga CJM, Pereira FV, et al. Anti-metastatic immunotherapy based on mucosal administration of flagellin and immunomodulatory P10. Immunol Cell Biol. 2015;93(1):86-98. https://doi.org/10.1038/ icb.2014.74.

128. Duong MTQ, Qin Y, You SH, Min JJ. Bacteria-cancer interactions: bacteria-based cancer therapy. Exp Mol Med. 2019;51(12):1. https://doi. org/10.1038/s12276-019-0297-0

129. Sasaki Y, Kojima A, Aoki H, Ogikubo Y, Takikawa N, Tamura Y. Phylogenetic analysis and PCR detection of Clostridium chauvoei, Clostridium haemolyticum, Clostridium novyi types A and B, and Clostridium septicum based on the flagellin gene. Vet Microbiol. 2002;86(3):257-67. https://doi.org/10.1016/S0378-1135(02)00002-0.

130. Rhee $\mathrm{SH}, \mathrm{Im} \mathrm{E}$, Pothoulakis $\mathrm{C}$. Toll-like receptor 5 engagement modulates tumor development and growth in a mouse xenograft model of human colon cancer. Gastroenterology. 2008;135(2):518-28. https://doi. org/10.1053/j.gastro.2008.04.022.

131. Faham A, Altin JG. Antigen-containing liposomes engrafted with flagellin-related peptides are effective vaccines that can induce potent antitumor immunity and immunotherapeutic effect. J Immunol. 2010;185(3):1744-54. https://doi.org/10.4049/jimmunol.1000027.

132. Roberts NJ, Zhang L, Janku F, et al. Intratumoral injection of Clostridium novyi-NT spores induces antitumor responses. Sci Transl Med. 2014;6(249):249ra111. https://doi.org/10.1126/scitranslmed.3008982.

133. Minton NP. Clostridia in cancer therapy. Nat Rev Microbiol. 2003;1(3):237-42. https://doi.org/10.1038/nrmicro777.

134. Catez F, Dalla Venezia N, Marcel V, Zorbas C, Lafontaine DU, Diaz Jل J. Ribosome biogenesis: an emerging druggable pathway for cance therapeutics. Biochem Pharmacol. 2018;2019(159):74-81. https://doi. org/10.1016/j.bcp.2018.11.014

135. Sveen K. The capacity of lipopolysaccharides from bacteroides, fusobacterium and veillonella to produce skin inflammation and the local and generalized Shwartzman reaction in rabbits. J Periodontal Res. 1977;12(5):340-50. https://doi.org/10.1111/j.1600-0765.1977.tb01525.x.

136. Hoshino K, Takeuchi O, Kawai T, et al. Pillars article: cutting edge: TollLike Receptor 4 (TLR4)-deficient mice are hyporesponsive to lipopolysaccharide: evidence for TLR4 as the Lps gene product. J Immunol. 1999;162:3749-52 J Immunol 2016 197(7) 25632566.http://www.ncbi. nlm.nih.gov/pubmed/27638938.

137. Tsukamoto H, Fukudome K, Takao S, Tsuneyoshi N, Kimoto M. Lipopolysaccharide-binding protein-mediated Toll-like receptor 4 dimerization enables rapid signal transduction against lipopolysaccharide stimulation on membrane-associated CD14-expressing cells. Int Immunol. 2010;22(4):271-80. https://doi.org/10.1093/intimm/dxq005.

138. Park GS, Kim JH. Myeloid differentiation primary response gene 88-leukotriene B4 receptor 2 cascade mediates lipopolysaccharide-potentiated invasiveness of breast cancer cells. Oncotarget. 2015;6(8):5749-59. https://doi.org/10.18632/oncotarget.3304.

139. Jain S, Dash P, Minz AP, et al. Lipopolysaccharide (LPS) enhances prostate cancer metastasis potentially through NF-kB activation and recurrent dexamethasone administration fails to suppress it in vivo. Prostate. 2019;79(2):168-82. https://doi.org/10.1002/pros.23722.

140. Li H, Xia J-Q, Zhu F-S, et al. LPS promotes the expression of PD-L1 in gastric cancer cells through NF-KB activation. J Cell Biochem. 2018;119:9997-10004. https://doi.org/10.1002/jcb.27329.
141. Deng YL, Tang DR, Hou PF, et al. Dysbiosis of gut microbiota in patients with esophageal cancer. Microb Pathog. 2020;2021(150): 104709. https://doi.org/10.1016/j.micpath.2020.104709.

142. Qi M, Liu J, Jiang Q, et al. Trueperella pyogenes pyolysin inhibits lipopolysaccharide-induced inflammatory response in endometrium stromal cells via autophagy- and ATF6-dependent mechanism. Brazilian J Microbiol. 2021;52(2):939-52. https://doi.org/10.1007/ s42770-021-00422-5.

143. Vettore $L$, Westbrook RL, Tennant DA. New aspects of amino acid metabolism in cancer. Br J Cancer. 2020;122(2):150-6. https://doi.org/ 10.1038/s41416-019-0620-5.

144. Wei Z, Liu X, Cheng C, Yu W, Yi P. Metabolism of amino acids in cancer Front Cell Dev Biol. 2021;8(January):603837. https://doi.org/10.3389/ fcell.2020.603837.

145. Ananieva E. Targeting amino acid metabolism in cancer growth and anti-tumor immune response. World J Biol Chem. 2015;6(4):281. https://doi.org/10.4331/wjbc.v6.i4.281.

146. Roomi MW, Ivanov V, Kalinovsky T, Niedzwiecki A, Rath M. Effect of ascorbic acid, lysine, proline, and green tea extract on human osteosarcoma cell line MNNG-HOS xenografts in nude mice: evaluation of tumor growth and immunohistochemistry. Med Oncol. 2006:23(3):411-7. https://doi.org/10.1385/MO:23:3:411.

147. Phillips MM, Sheaff MT, Szlosarek PW. Targeting arginine-dependent cancers with arginine-degrading enzymes: opportunities and challenges. Cancer Res Treat. 2013;45(4):251-62. https://doi.org/10.4143/ crt.2013.45.4.251.

148. Grzywa TM, Sosnowska A, Matryba P, et al. Myeloid cell-derived arginase in cancer immune response. Front Immunol. 2020;11:938. https:// doi.org/10.3389/fimmu.2020.00938.

149. Kertys M, Grendar M, Horak V, Zidekova N, Kupcova Skalnikova H, Mokry J, Halasova E, Strnadel J. Metabolomic characterisation of progression and spontaneous regression of melanoma in the melanoma-bearing Libechov minipig model. Melanoma Res. 2021;31(2):140-51. https://doi.org/10.1097/CMR.0000000000 000722.

150. de Ingeniis J, Ratnikov B, Richardson AD, et al. Functional specialization in proline biosynthesis of melanoma. PLoS One. 2012;7(9):1-9. https://doi.org/10.1371/journal.pone.0045190.

151. Kardos GR, Wastyk HC, Robertson GP. Disruption of proline synthesis in melanoma inhibits protein production mediated by the GCN2 pathway. Mol Cancer Res. 2015;13(10):1408-20. https://doi.org/10. 1158/1541-7786.MCR-15-0048.

152. Phang JM. Proline metabolism in cell regulation and cancer biology: recent advances and hypotheses. Antioxidants Redox Signal. 2019;30(4):635-49. https://doi.org/10.1089/ars.2017.7350.

153. Siddiqui A, Ceppi P. A non-proliferative role of pyrimidine metabolism in cancer. Mol Metab. 2020;35(2): 100962. https://doi.org/10.1016/j. molmet.2020.02.005

154. Wang W, Cui J, Ma H, Lu W, Huang J. Targeting pyrimidine metabolism in the era of precision cancer medicine. Front Oncol. 2021;11(5):1-17. https://doi.org/10.3389/fonc.2021.684961.

155. Wang H, Wang $X, X u L$, Zhang J, Cao H. High expression levels of pyrimidine metabolic rate-limiting enzymes are adverse prognostic factors in lung adenocarcinoma: a study based on the cancer genome atlas and gene expression omnibus datasets. Purinergic Signal. 2020;16(3):347-66. https://doi.org/10.1007/s11302-020-09711-4.

156. Galmarini $C M$, Jordheim $L$, Dumontet C. Pyrimidine nucleoside analogs in cancer treatment. Expert Rev Anticancer Ther. 2003;3(5):71728. https://doi.org/10.1586/14737140.3.5.717.

157. Christman JK. 5-Azacytidine and 5-aza-2'-deoxycytidine as inhibitors of DNA methylation: mechanistic studies and their implications for cancer therapy. Oncogene. 2002;21(35):5483-95. https://doi.org/10. 1038/sj.onc.1205699.

158. Yin J, Ren W, Huang X, Deng J, Li T, Yin Y. Potential mechanisms connecting purine metabolism and cancer therapy. Front Immunol. 2018:9(July):1-8. https://doi.org/10.3389/fimmu.2018.01697.

159. Su WJ, Lu PZ, Wu Y, Kalpana K, Yang CK, Lu GD. Identification of key genes in purine metabolism as prognostic biomarker for hepatocellular carcinoma. Front Oncol. 2021;10(January):1-13. https://doi.org/10. 3389/fonc. 2020.583053 
160. Song J, Zhang Y, Zhang C, et al. A microfluidic device for studying chemotaxis mechanism of bacterial cancer targeting. Sci Rep. 2018:8(1):1-9. https://doi.org/10.1038/s41598-018-24748-7.

161. Chen Y, Li P. Fatty acid metabolism and cancer development. Science Bulletin. 2016;61(19):1473-9. https://doi.org/10.1007/ s11434-016-1129-4.

162. Röhrig F, Schulze A. The multifaceted roles of fatty acid synthesis in cancer. Nat Rev Cancer. 2016;16(11):732-49. https://doi.org/10.1038/ nrc.2016.89.

163. Koundouros N, Poulogiannis G. Reprogramming of fatty acid metabolism in cancer. Br J Cancer. 2020;122(1):4-22. https://doi.org/10.1038/ s41416-019-0650-z.

164. Muinonen-Martin AJ, Susanto O, Zhang Q, et al. Melanoma cells break down LPA to establish local gradients that drive chemotactic dispersal. PLoS Biol. 2014;12(10): e1001966. https://doi.org/10.1371/journal.pbio. 1001966.

165. Saksena S, Dudeja PK. Role of membrane transporters in intestinal cancers. Cmgh. 2019;7(1):241-2. https://doi.org/10.1016/j.jcmgh.2018.09.016.

166. Yarwood R, Hellicar J, Woodman PG, Lowe M. Membrane trafficking in health and disease. DMM Dis Model Mech. 2020;13(4):dmm043448. https://doi.org/10.1242/dmm.043448.

167. Samuelson DR, Welsh DA, Shellito JE. Regulation of lung immunity and host defense by the intestinal microbiota. Front Microbiol. 2015:6(OCT):1-14. https://doi.org/10.3389/fmicb.2015.01085.

168. Komiya Y, Shimomura Y, Higurashi T, et al. Patients with colorectal cancer have identical strains of Fusobacterium nucleatum in their colorectal cancer and oral cavity. Gut. 2019;68(7):1335-7. https://doi.org/10. 1136/gutjnl-2018-316661.

169. Abed J, Maalouf N, Manson AL, et al. Colon cancer-associated Fusobacterium nucleatum may originate from the oral cavity and reach colon tumors via the circulatory system. Front Cell Infect Microbiol. 2020;10(August):1-12. https://doi.org/10.3389/fcimb.2020.00400.

170. Bullman S, Pedamallu CS, Sicinska E, et al. Analysis of Fusobacterium persistence and antibiotic response in colorectal cancer. Science. 2017:358(6369):1443-8. https://doi.org/10.1126/science.aal5240.

171. Bennett KW, Eley A. Fusobacteria: new taxonomy and related diseases J Med Microbiol. 1993;39(4):246-54. https://doi.org/10.1099/00222 615-39-4-246.

172. Darenfed H, Grenier D, Mayrand D. Acquisition of plasmin activity by Fusobacterium nucleatum subsp, nucleatum and potential contribution to tissue destruction during periodontitis. Infect Immun. 1999:67(12):6439-44. https://doi.org/10.1128/iai.67.12.6439-6444.1999.

173. Wang S, Liu Y, Li J, et al. Fusobacterium nucleatum acts as a pro-carcinogenic bacterium in colorectal cancer: from association to causality. Front Cell Dev Biol. 2021;9(August):1-15. https://doi.org/10.3389/fcell. 2021.710165

174. Pignatelli P, lezzi $L$, Pennese $M$, et al. The potential of colonic tumor tissue fusobacterium nucleatum to predict staging and its interplay with oral abundance in colon cancer patients. Cancers (Basel). 2021:13(5):119. https://doi.org/10.3390/cancers13051032.

175. Abed J, Emgård JEM, Zamir G, et al. Fap2 mediates Fusobacterium nucleatum colorectal adenocarcinoma enrichment by binding to tumor-expressed Gal-GalNAc. Cell Host Microbe. 2016;20(2):215-25. https://doi.org/10.1016/j.chom.2016.07.006.

176. Fliegerova K, Tapio I, Bonin A, et al. Effect of DNA extraction and sample preservation method on rumen bacterial population. Anaerobe. 2014;29:80-4. https://doi.org/10.1016/j.anaerobe.2013.09.015.

177. Bolyen E, Rideout JR, Dillon MR, et al. Reproducible, interactive, scalable and extensible microbiome data science using QIIME 2. Nat Biotechnol. 2019:37(8):852-7. https://doi.org/10.1038/s41587-019-0209-9.

178. Callahan BJ, McMurdie PJ, Rosen MJ, Han AW, Johnson AJA, Holmes SP. DADA2: high-resolution sample inference from Illumina amplicon data. Nat Methods. 2016;13(7):581-3. https://doi.org/10.1038/nmeth. 3869.

179. Katoh K, Misawa K, Kuma KI, Miyata T. MAFFT: a novel method for rapid multiple sequence alignment based on fast fourier transform. Nucleic Acids Res. 2002;30(14):3059-66. https://doi.org/10.1093/nar/ gkf436.

180. Price MN, Dehal PS, Arkin AP. FastTree 2 - approximately maximumlikelihood trees for large alignments. PLoS One. 2010;5(3):e9490. https:// doi.org/10.1371/journal.pone.0009490.
181. Rognes T, Flouri T, Nichols B, Quince C, Mahé F. VSEARCH: a versatile open source tool for metagenomics. PeerJ. 2016;2016(10):1-22. https:// doi.org/10.7717/peerj.2584.

182. RStudio Team. RStudio: integrated development for R. Boston MA: RStudio PBC; 2020. (http://www.rstudio.com/)

183. Jordan E Bisanz (2018) qiime2R: Importing QIIME2 artifacts and associated data into R sessions. https://github.com/jbisanz/qiime2R.

184. Wickham H. ggplot2: Elegant Graphics for Data Analysis. SpringerVerlag New York. ISBN 978-3-319-24277-4, https://ggplot2.tidyverse. org.

185. McMurdie PJ, Holmes S. Phyloseq: an R package for reproducible interactive analysis and graphics of microbiome census data. PLoS One. 2013:8(4):e61217.

186. Segata N, Izard J, Waldron L, et al. Metagenomic biomarker discovery and explanation. Genome Biol. 2011;12(6):1-18. https://doi.org/10. 1186/gb-2011-12-6-r60

187. Langille MGI, Zaneveld J, Caporaso JG, et al. Predictive functional profiling of microbial communities using $16 \mathrm{~S}$ rRNA marker gene sequences. Nat Biotechnol. 2013;31(9):814-21. https://doi.org/10.1038/nbt.2676.

188. Parks DH, Tyson GW, Hugenholtz P, Beiko RG. STAMP: Statistical analysis of taxonomic and functional profiles. Bioinformatics. 2014;30(21):31234. https://doi.org/10.1093/bioinformatics/btu494.

\section{Publisher's Note}

Springer Nature remains neutral with regard to jurisdictional claims in published maps and institutional affiliations.

Ready to submit your research? Choose BMC and benefit from

- fast, convenient online submission

- thorough peer review by experienced researchers in your field

- rapid publication on acceptance

- support for research data, including large and complex data types

- gold Open Access which fosters wider collaboration and increased citations

- maximum visibility for your research: over $100 \mathrm{M}$ website views per year

At BMC, research is always in progress.

Learn more biomedcentral.com/submissions 